\title{
Intrusions into the shadow of attention: A new take on illusory conjunctions
}

\author{
Cynthia M. Henderson ${ }^{1} \cdot$ James L. McClelland ${ }^{1}$
}

Published online: 18 March 2020

(C) The Psychonomic Society, Inc. 2020

\begin{abstract}
We present new evidence about illusory conjunctions (ICs) suggesting that their current explanation requires revision. According to Feature Integration Theory (FIT; Treisman \& Gelade Cognitive Psychology, 12, 97-136, 1980), focal attention to a single stimulus is required to bind its features into an integrated percept. FIT predicts that if attention is spread over multiple stimuli, features of these different stimuli can be combined into a single percept and produce ICs. Treisman and Schmidt (Cognitive Psychology, 14, 107-141, 1982) and Cohen \& Ivry (Journal of Experimental Psychology: Human Perception and Performance, 15(4), 650-663, 1989) supported this prediction. In the latter study, participants viewed brief displays containing two digits and two colored letters. Digit locations were pre-cued, and participants were instructed to prioritize the digits and to spread their attention across the region encompassed by the digits. Cohen \& Ivry found that reports of one letter (the 'target') produced ICs when both letters appeared between the digits. Expanding on Cohen \& Ivry's paradigm, we find that both letters do not need to appear between the digits to produce ICs. While the target letter was highly susceptible to ICs if the target appeared inside the position of a nearby digit, the position of the other letter was largely irrelevant. Our experimental results also argue that these ICs were not due to mnemonic errors occurring while the digits are being reported. Based on our findings, we propose that attention to the digits casts an attentional 'shadow' projecting towards fixation, interfering with processing of target letters in that shadow and allowing color information from elsewhere in the display to be included in the resulting percept.
\end{abstract}

Keywords Attention: Object based $\cdot$ Illusory conjunctions $\cdot$ Feature integration theory $\cdot$ Vision: Spatial attention

Illusory conjunction (IC) errors occur when a participant reports a stimulus that incorrectly combines the features of two presented stimuli. These errors, famously described by Treisman \& Gelade (1980), motivated an important theory of attention called Feature Integration Theory (FIT). However, despite numerous studies on ICs (see e.g., Pelli et al., 2004), the necessary conditions for producing ICs have been unclear. Several of our initial experiments failed to produce an IC effect despite adhering to the conditions thought

Electronic supplementary material The online version of this article (https://doi.org/10.3758/s13414-019-01893-3) contains supplementary material, which is available to authorized users.

Cynthia M. Henderson

cynthia.henderson26@gmail.com

James L. McClelland

jlmcc@stanford.edu

1 Stanford University, Stanford, CA, USA necessary to produce ICs according to FIT. It was not until we strictly replicated the experiment of Cohen \& Ivry (1989) that we produced ICs reliably. In this and another paper (Henderson, 2014), we describe our insights into the conditions we found for producing ICs, and how these conditions relate to predictions by FIT.

In this paper and in Henderson (2014), we argue that two types of ICs have been conflated in the literature. In the first case, and representing the majority of IC effects documented in the literature, ICs occur between closely neighboring target and distractor stimuli. Henderson (2014) argued that these proximal IC errors can be largely or entirely explained by the crowding effect, a limitation of visual perception such that stimuli placed within a certain critical spacing of each other will interfere with each other's perception (Whitney \& Levi, 2011; Pelli \&Tillman, 2008; see also Pelli et al., 2004).

A second, smaller set of studies describe ICs between distal stimuli (e.g., Cohen \& Ivry, 1989). We define distal ICs as IC effects between two stimuli that are spaced far enough apart that neither is crowding the other. For the purposes of this 
paper, distal ICs are particularly intriguing in that they could possibly serve as evidence for FIT. Furthermore, without FIT, it is not clear what mechanisms would lead to interference on a target stimulus from a distant stimulus positioned as far away as the opposite visual hemifield, especially considering widespread evidence that most early visual processing is carried out largely independently for different spatial regions by small, localized receptive fields. In this paper, we focus on these distal ICs and examine the conditions required to produce them. As we shall describe, our findings challenge aspects of FIT. Taken together with other findings, the evidence we will report supports an alternative conception of the role of visual attention in the identification of items in a display, as we will explain in the general discussion.

\section{Review}

Feature Integration Theory (Treisman \& Gelade, 1980) provided early theoretical backing for ICs. Under FIT, ICs represent a type of error in the perceptual binding of features when attention is diverted. According to FIT, the stimulus characteristics at a given position are bound together into a perceived object when an attentional spotlight focuses on that spatial position. If multiple items appear within the visual field, visual attention must shift to each item in turn to perceive them properly. When the items are presented very briefly or sequential attention to each item's location is otherwise prevented, FIT predicts that the visual system could group features into perceived stimuli at random and therefore may potentially produce ICs.

Work by Anne Treisman and colleagues motivated many of the early studies to successfully demonstrate ICs. These IC studies appear to fall into two groups: In the first group of studies, the stimuli which produce ICs appear in close proximity to each other relative to their distance from fixation. For example, Prinzmetal, Henderson, \& Ivry (1995) positioned a closely spaced string of four letters in the upper right portion of a display. The string consisted of two colored letters (an ' $\mathrm{X}$ ' and an ' $\mathrm{O}$ ') flanked by two white 'O's. Participants were asked to report the non- ' $\mathrm{O}$ ' colored letter, and it was considered an IC error when participants correctly identified the target letter (X, T, or L) but reported the color of the distractor ' $\mathrm{O}$ ' instead of the actual color of the target letter.

Prinzmetal, Henderson, \& Ivry (1995) demonstrated that ICs occur under these proximal conditions. Furthermore, stimulus duration and dual task conditions did not affect these ICs. Participants produced substantial ICs regardless of whether they simultaneously completed an engaging RSVP task, and ICs were similarly present when stimulus duration was set either to a short $(150 \mathrm{~ms})$ or long $(15,000 \mathrm{~ms})$ duration.

Other experiments that found ICs with tightly spaced stimuli suggest that the proximity of the stimuli appears to be critical. Numerous studies have found ICs between stimuli at close proximities, with evidence that ICs decreased as the stimuli were placed farther from each other (Ashby, Prinzmetal, Ivry, \& Maddox, 1996; Chastain, 1982; Ivry \& Prinzmetal, 1991; Keele et al., 1988; Prinzmetal \& Keysar, 1989; Prinzmetal \& Mills-Wright, 1984; Prinzmetal, Treiman, \& Rho, 1986; Wolford \& Shum, 1980). Henderson (2014) in particular found that these proximal ICs disappeared when the distance between stimuli increased beyond a certain point.

We note that each of these proximal IC results occurred with the distractor stimulus positioned within the critical crowding distance of the target stimulus, roughly $40-70 \%$ of the target stimulus' eccentricity (Bouma, 1970; Whitney \& Levi, 2011; Vickery et al., 2009). Pelli et al. (2004) suggested that these IC effects may be related to crowding, and in Henderson (2014) these proximal ICs exhibited several definitive characteristics of crowding, including a critical spacing, a radial-tangential anisotropy, an upper-lower visual field bias, and an effect of the number of flankers. These findings argue that many cases of illusory conjunctions may be a consequence of spatially constrained visual processing rather than evidence of limitations in feature binding.

In contrast to these proximal IC experiments, several IC results cannot be explained by crowding. What we term as distal ICs have been found with interstimulus spacings of 100-200\% of a target stimulus' eccentricity, including several cases in which the source of the intrusion error is in the hemifield opposite to the target (Treisman \& Schmidt, 1982; Cohen \& Ivry, 1989; Navon \& Ehrlich, 1995; Tsal, 1989). As an additional distinction between these types of ICs, distal ICs also appear to require a more restrictive set of experimental conditions than proximal ICs. Whereas proximal ICs occur even with long stimulus durations and no dual task (e.g., Prinzmetal, Henderson, \& Ivry, 1995), distal ICs have typically been found under dual-task conditions with short stimulus durations and a post-stimulus mask.

In exploring distal ICs, we focus on an experimental design used by Treisman \& Schmidt (1982) and Cohen \& Ivry (1989) that has provided valuable insights into the mechanisms and parameters of distal ICs.

Treisman \& Schmidt (1982) helped establish the existence of ICs as evidence for Feature Integration Theory. In their Experiment 4, four colored stimuli were presented in two rows at the center of an image and were flanked by a black digit on each side. The four potential target stimuli varied in their features: they could be circles or triangles, filled or hollow, and large or small; similarly, each of the four stimuli had a distinct color. The image was presented for a short duration and followed by a cue at the location of one of the four stimuli.

After viewing the stimuli, participants were asked to identify the digits and then the target stimulus. Participants had been instructed that identifying the black digits was their priority and were asked to spread their attention across the digits (and therefore the colored stimuli between them). Reporting the features of the cued stimulus was their secondary priority in this task. 
Participant's responses were coded as conjunction errors if they reported a feature from a non-cued stimulus, and were considered feature errors if they reported a feature that was not present on that trial. Feature errors were used to control for guessing; an IC was determined to have occurred if participants had more conjunction errors than feature errors.

Treisman \& Schmidt predicted that these colored stimuli would be susceptible to IC errors to the extent that the digit task succeeded in spreading an attentional spotlight across several of the stimuli and prevented participants from focusing attention on individual colored stimuli. Consistent with this, Treisman \& Schmidt found a significant IC effect across several experiments in which the prioritized digit report was required, and the IC effect disappeared when participants no longer performed the digit task (see their Experiment 5).

While these findings helped bring ICs into the spotlight, several researchers have disputed their conclusions. The lack of ICs in the non-digit condition may be alternately explained by the lower attentional, visual, and mnemonic demands when the digits and digit task were removed (see e.g., Tsal, 1989; Navon \& Ehrlich, 1995). In addition, Treisman \& Schmidt's non-digit experiment pre-cued the location of the target stimulus, changing the nature of the task from identification of a target among similar distractors to identification of a briefly presented target with a pre-cued location.

Cohen \& Ivry (1989) addressed several concerns about the role of the digit task from Treisman \& Schmidt (1982). Rather than manipulating the presence or absence of dual task conditions, Cohen \& Ivry measured ICs while varying whether the potential target stimuli both appeared within the attended region bounded by the digits. Cohen \& Ivry used a pair of digits as primary task stimuli and a pair of colored letters as the target and distractor (Fig. 1). The experimenters prompted participants to expand their attentional spotlight over a portion of their visual field by pre-cueing the locations of the digitswhich participants had to report as their top priority - and then manipulated whether the target (a colored letter) and distractor stimuli (always the letter ' $\mathrm{O}$ ', in a different color that the target letter) appeared between those digits. A participant's response would be coded as an IC if they report the identity of the target letter correctly but report the color of the distractor stimulus. Cohen \& Ivry found that ICs mainly occurred when both stimuli appeared in the attended region. If either the target or the distractor stimulus instead appeared outside the positions of the digits, the IC effect was weak or absent. These results by Cohen \& Ivry support an FIT-inspired mechanism in which only those features within the spotlight are available for miscombination, with the caveat that the penumbra of the spotlight might extend beyond the positions of the cues.

We began our experiments by replicating the main findings of Cohen \& Ivry (1989; Experiments 3-4). Additional analyses revealed that ICs were highly driven by the position of the target stimulus relative to the nearest digit, and were largely independent of distractor position. Target accuracy was lower and ICs were more prevalent when the target appeared just inside the location of a nearby digit, relative to fixation, while IC rates were unaffected by whether the distractor stimulus appeared between the digits or not. We term this as the 'inner-outer effect', and found it across all of our experiments where distal ICs occurred.

Experiment 2 revisited the role of the digit task in producing ICs as explored in Treisman \& Schmidt's (1982) non-digit experiment. Experiment 2 removed the dual-task requirements but retained visual presentation of the digits, exploring whether visual presentation of the distractor stimuli alone could produce ICs or the inner-outer effect, particularly for target accuracy.

Experiments 3-5 explored questions raised by Navon \& Ehrlich (1995), who considered whether response order may drive ICs. Navon \& Ehrlich argued that ICs may be related to mnemonic errors from only reporting target stimuli after the digits (see also Tsal, 1989). Experiment 3 investigated this question by switching the order of responses to report the target letter before the digits. We found that the IC effect was eliminated under these conditions. However, it was unclear if the IC effect disappeared due to the response order alone or due to reduced prioritization of the digits resulting from the changed response order. To facilitate examination of this question, Experiment 4 boosted our statistical power by increasing the number of trials per condition, while Experiment 5 introduced a post-stimulus cue to indicate response order on each trial after the stimulus had been viewed. Experiment 5 found an IC effect that was attenuated but not eliminated when the target letter was reported first, arguing that ICs cannot be solely attributed to mnemonic influences.

In summary, our experiments clarify and extend the conditions under which distal ICs can be obtained. Certain results, particularly the inner-outer effect, suggest some revision of the FIT account of ICs may be necessary. We continue discussion of how our results might be interpreted in the General Discussion.

\section{Experiment 1}

Experiment 1 replicated the conditions of Cohen \& Ivry (1989; Experiments 3-4), a dual-task experiment in which participants identified two briefly presented digits ("competing task stimuli') and a target letter while ignoring a distractor letter. The design encouraged preferential attention to the digits, as the digits' locations were pre-cued, they appeared slightly prior to the target, and they were reported before reporting on the target. The positions of these pre-cued digits were used to manipulate the spread of the attentional spotlight for each trial. Cohen \& Ivry predicted that ICs would occur when the target and distractor letters both fell within this spotlight of attention. 


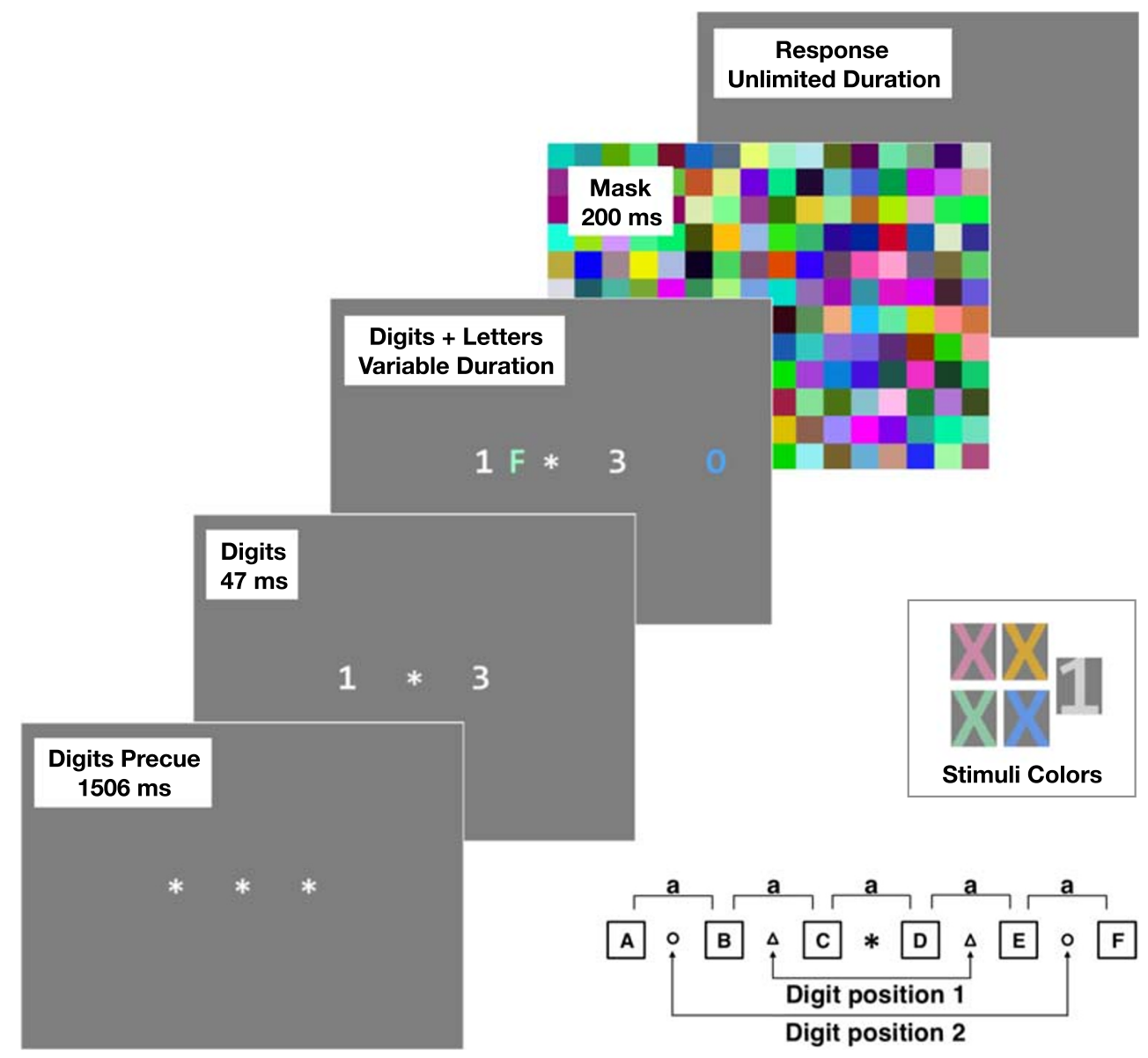

Fig. 1 Example depiction of a trial in an illusory conjunction task. This reflects the experimental setup in this paper's Experiment 1 and closely matches that of Cohen \& Ivry's (1989) Experiment 3. Colors enhanced for visibility; see inset labeled Stimuli Colors for actual colors used (RGB luminance values provided in the Supplementary Materials). Diagram (bottom right) depicts the positions at which stimuli could appear. Digits appeared either at the positions of the triangles $\left( \pm 1.6^{\circ}\right.$; 'Small

Supporting this, the authors found illusory conjunctions when the target and distractor letters appeared between the two digits. However, they also found ICs when the letters were outside and immediately adjacent to the digits, a result they attributed to an imprecise spread of the spotlight of attention.

Our Experiment 1 found a similar pattern of results, but further analyses revealed details that are hard to explain under an attentional spotlight version of ICs.

\section{Participants}

Eighteen participants from the Stanford Subject Pool participated in an hour-long experiment. Two participants received course credit and the others were paid $\$ 12$ for their participation. None of the participants reported being color blind.
Spotlight') or circles $\left( \pm 3.2^{\circ}\right.$; 'Large Spotlight'). Letters could appear in positions A, B, C, D, E, or F. On each trial, one letter appeared to the left of fixation while the other appeared to the right. Boxes around letters are used for diagrammatic purposes and were not present in the display. The figure depicts Experiments 1, 2, and 3; for Experiments 4 and 5, letter positions A and F were not used. (a): Distance between letters: $1.6^{\circ}$

\section{Stimuli}

Stimuli were presented on a HP p1230 CRT monitor with an $85-\mathrm{Hz}$ refresh rate, at a distance of approximately $1.15 \mathrm{~m}$ from the participant (further details in Supplementary Materials)

Stimuli consisted of a fixation cue, pre-cues in the shape of white asterisks; white digits ranging from 1 to 4; and colored letters (X, F, or $\mathrm{O}$ ), which could be pink, yellow, green, or blue. All stimuli were presented on a gray background (Fig. 1).

Each trial involved two digits and two colored letters. One colored letter was always an ' $\mathrm{O}$ ' (the distractor) and the other could be either an ' $\mathrm{X}$ ' or an ' $\mathrm{F}$ ' (the target). The two digits always had different values and the two letters always had different colors.

The digits and letters had a height of $0.6^{\circ}$ and maximum width of $0.48^{\circ}$. Digits and letters were arranged on a horizontal axis with up to $4.0^{\circ}$ offset from fixation (Fig. 1). Digit 
displacement was $1.6^{\circ}$ for the small spotlight condition and $3.2^{\circ}$ for the large spotlight conditions, while the two letters could have a displacement of $0.8^{\circ}, 2.4^{\circ}$, or $4.0^{\circ}$. For comparison with crowding effects, it has often been found that target stimuli are crowded by flankers appearing within $50 \%$ of the target stimulus' eccentricity (Bouma, 1970), though the exact coefficient can vary between studies, based on factors such as the attentional demands of the task (Whitney \& Levi, 2011). Digits in the small spotlight condition are within the critical spacing to crowd letters in positions $\mathrm{B}$ and $\mathrm{E}$. For the large spotlight condition, letters at positions $\mathrm{A}, \mathrm{B}, \mathrm{E}$, and $\mathrm{F}$ would be susceptible to crowding from digit stimuli ${ }^{1}$.

Stimuli were followed by a mask, which consisted of $12 \times 24$ tiles of colored squares for a total size of $7.5^{\circ} \times 15^{\circ}$. The color of each square was randomly generated.

While the experiment was created to match Cohen \& Ivry's (1989) Experiment 3, later discussion revealed two main differences. First, we added a fixation mark at the center of the screen. Second, our background color was gray rather than black (R. Ivry, pers. comm., January 16, 2012).

\section{Procedure}

Each trial began with a fixation mark and pre-cues for the digits' locations, which appeared for $1506 \mathrm{~ms}$ (Fig. 1). The pre-cues were replaced by digits for $47 \mathrm{~ms}$, after which they were joined on the screen by the two colored letters. The duration of the digit+letter display was determined by an adaptive procedure during practice trials (see Supplementary Materials for details). Following the digit and letter display, a mask was presented for $200 \mathrm{~ms}$, and then a blank screen appeared for participant reports. Participants were instructed to report both digits before reporting the color and identity of the target letter, which they were told was always either an X or an F. The order in which digits were reported did not matter. Responses were self-paced with no time limit. The inter-trial interval was $1000 \mathrm{~ms}$.

There were three blocks of test trials with 72 trials per block. Letter and digit eccentricities were counterbalanced on each block of trials, as were the target letter's identity and whether it appeared to the left or right of fixation. The distractor letter always appeared on the side opposite the target letter.

Participants were instructed multiple times that identifying the digits was their primary goal on each trial.

\footnotetext{
${ }^{1}$ Data from Experiment 2 suggest it is unlikely that crowding underlies our distal IC results, as the IC effect disappeared when participants no longer attended to the digits. In contrast, crowding is found in the absence of attention to the flanking stimuli.
}

\section{Data analysis}

We used a measure of illusory conjunctions that corrects for the possibility that the participant simply guessed the color of the distractor letter. Illusory conjunctions are considered to have occurred if the frequency of conjunction errors (where the target letter's identity is correctly reported, but with the distractor letter's color) exceeds the frequency expected by random guessing, as estimated by the frequency of color errors (where the target letter's identity is correctly reported, but with a color that did not appear in that trial). For our initial results, we use only trials in which the participant correctly reported the target letter identity (' $\mathrm{X}$ ' or ' $\mathrm{F}$ '). If participants were simply guessing the target letter's color when they failed to correctly perceive it, $1 / 4$ of the time they would guess the correct color, $1 / 4$ of the time they would guess the distractor letter's color, and $1 / 2$ of the time they would guess a color that was not present on that trial. Based on this, in the absence of a true IC effect, there should be half as many conjunction errors as there are color errors. Thus, we estimate the IC rate using the following formula:

\section{Est. IC Rate $=$ Conjunction Errors $-(0.5 \times$ Color Errors $)$}

A more sophisticated method of measuring ICs exists (Ashby et al., 1996). However, it requires estimation of additional error types, which were unstable due to their low incidence rates. We use this simpler method as a compromise.

We note that our measure likely underestimates the IC effect: Cases when a participant has neither a conjunction error nor a color error are counted as evidence for the null hypothesis, when more aptly they might be counted as providing no information ${ }^{2}$.

Like Cohen \& Ivry (1989), we combined certain symmetric positions such as $\mathrm{BD}$ and $\mathrm{CE}$ to compensate for the low number of trials (see Fig. 1). Given our a priori hypotheses about the direction of effects, these analyses are often one-tailed. Furthermore, they were not corrected for multiple comparisons due to concerns about Type II errors with the low incidence of IC and color errors in each condition. Rather, it is hoped that consistency in certain effects can be found across the multiple similar experiments reported in this paper.

\section{Results and discussion}

Experiment 1 attempted to replicate the findings of Experiments 3 and 4 from Cohen \& Ivry (1989). Similar to Cohen \& Ivry, we found a significant IC effect in our experiment: The estimated IC rate significantly exceeded the value expected by random guessing, $\mathrm{t}(17)=2.23, p<0.05$ (see Table 1). In addition, our adaptive

\footnotetext{
${ }^{2}$ That is, our results compare the estimated IC rate of a set of subjects against the null hypothesis of 0 . Participants who had neither conjunction errors nor color errors in a condition would have an estimated IC rate of 0 , indistinguishable from participants who had an equal number of conjunction and feature errors.
} 
Table 1 Means of various measures in Cohen \& Ivry's (1989) Experiment 3 and our Experiments 1-5

\begin{tabular}{|c|c|c|c|c|c|c|c|}
\hline Experiment & $C \& I$ & 1 & 2 & 3 & 4 & $5-\mathrm{D}$ & $5-\mathrm{L}$ \\
\hline Digit + letter duration (ms) & 118 & $128.7(25.3)$ & $62.3(11.6)$ & $146.4(52.0)$ & $142.6(13.7)$ & $152.9(7.6)$ & $152.9(7.6)$ \\
\hline Digit accuracy & $92.0 \%$ & $92.5 \%(4.3 \%)$ & NA & $83.1 \%(12.9 \%)$ & $95.8 \%(3.3 \%)$ & $87.6 \%(10.1 \%)$ & $75.3 \%(19.5 \%)$ \\
\hline Letter accuracy & $54.0 \%$ & $78.9 \%(14.8 \%)$ & $80.0 \%(10.6 \%)$ & $86.7 \%(12.3 \%)$ & $84.7 \%(12.1 \%)$ & $72.8 \%(17.7 \%)$ & $81.9 \%(15.9 \%)$ \\
\hline Conjunction error & $11.6 \%$ & $7.9 \%(8.8 \%)$ & $4.2 \%(3.1 \%)$ & $2.6 \%(2.6 \%)$ & $6.1 \%(6.8 \%)$ & $9.4 \%(6.5 \%)$ & $5.4 \%(5.5 \%)$ \\
\hline Color error & $16.3 \%$ & $5.9 \%(3.5 \%)$ & $10.6 \%(4.5 \%)$ & $4.5 \%(4.9 \%)$ & $3.3 \%(3.3 \%)$ & $3.9 \%(4.4 \%)$ & $2.9 \%(2.9 \%)$ \\
\hline Letter ID error & $8.2 \%$ & $4.5 \%(3.4 \%)$ & $3.0 \%(2.1 \%)$ & $4.0 \%(3.8 \%)$ & $4.0 \%(3.7 \%)$ & $7.8 \%(6.5 \%)$ & $6.1 \%(5.9 \%)$ \\
\hline Letter ID + conjunction error & $4.5 \%$ & $1.9 \%(3.7 \%)$ & $0.9 \%(1.2 \%)$ & $0.8 \%(1.5 \%)$ & $0.9 \%(1.0 \%)$ & $3.8 \%(5.2 \%)$ & $2.3 \%(3.4 \%)$ \\
\hline Letter ID + color error & $5.4 \%$ & $1.0 \%(1.3 \%)$ & $1.3 \%(1.5 \%)$ & $1.5 \%(2.3 \%)$ & $1.1 \%(1.5 \%)$ & $2.3 \%(2.3 \%)$ & $1.4 \%(2.5 \%)$ \\
\hline
\end{tabular}

Note. Standard deviation values are in parentheses. For comparison against Cohen \& Ivry (1989), these values include only digit-correct trials. 5-D = digit first, $5-\mathrm{L}=$ letter first

mechanism to control digit accuracy was similarly effective to Experiment 3 of Cohen \& Ivry, with no significant difference in digit accuracy between their experiments and ours in either the small or large spotlight conditions, $\mathrm{t}(17)=0.6$ and $1.4, p>0.10$ (Table 1).

However, our results differed from those of Cohen \& Ivry (1989) on two important measures. We found a stronger IC effect than Cohen \& Ivry: When we compared the total number of conjunction and color errors in our experiment against the conjunction and color errors for either Experiment 3 or Experiment 4 in Cohen \& Ivry using a $\chi^{2}$ test of independence, we found that our experiment had a higher proportion of conjunction-to-color errors, $\chi^{2}(\mathrm{df}=1)>16.3, p<0.001$. In addition, target letter accuracy was significantly greater than the value reported in Cohen \& Ivry's Experiment 3 across both the small and large spotlight conditions, $\mathrm{t}(17)=5.9$ and $9.5, p$ $<0.001$.

These differences may be related to our use of a gray background rather than the black background in Cohen \& Ivry. Three similar exploratory studies with a total of 23 participants used a black background with slight variations in the letter colors. Similar to Cohen \& Ivry's results, participants in these studies had a lower target accuracy than participants in our Experiment 1, $\mathrm{M}=54.2 \%(\mathrm{SD}=16.3 \%), \mathrm{t}(39)=5.0, p<$ 0.001 . These participants also had a lower ratio of IC-to-color errors, interaction effect in two-way ANOVA F $(1,78)=14.8$, $p<0.001$.

Subjectively speaking, we felt that the colors looked more similar on the black background than on the gray background. Increased similarity in experiments with a black background may have reduced discriminability between colors and led to more random guessing, decreasing both target accuracy and the IC effect (see e.g., Ivry \& Prinzmetal, 1991).

These increases in the overall magnitude of our IC effect likely contributed to our finding of IC effects in positions beyond those of Cohen \& Ivry (1989). We compare our experimental results against Cohen \& Ivry's Experiment 3 rather than Experiment 4 as it is was a closer match to both our inter-stimulus distances as well as the stimulus duration for small and large spotlight conditions; however, both had similar results. Cohen \& Ivry's Experiment 3 found significant ICs for letter positions $\mathrm{CD}$ in the small spotlight, and $\mathrm{CD}, \mathrm{BD}-$ $\mathrm{CE}, \mathrm{AD}-\mathrm{CF}$ in the large spotlight condition, while $\mathrm{BE}$ approached significance (see Fig. 1 and the Supplementary Materials). Consistent with the idea that ICs occur when the letters fall within an attentional spotlight generated by the digits, almost all of their ICs occurred when the letters appeared between the digits.

In comparison, we found significant IC effects even when the letters were well outside the perimeter of the digits. For the small spotlight condition, only in positions CD did the letters appear between the digits. However, we found significant IC effects for positions $\mathrm{CD}, \mathrm{BD}-\mathrm{CE}$, and $\mathrm{AE}-\mathrm{BF}, \mathrm{t}(17)=3.1$, 2.5, and 2.1, $p<0.05$, while position AD-CF approached significance, $\mathrm{t}=1.9, p<0.10$ (Table 1 ).

Our results were more similar to Cohen \& Ivry's in the large spotlight condition, where they found that positions $\mathrm{BD}-\mathrm{CE}$ and BE had significant IC effects, $\mathrm{t}(17)=3.1$ and $2.6, p<0.05$, and there was a marginally significant IC effect for position $\mathrm{AD}-\mathrm{CF}, \mathrm{t}=2.1, p<0.10$.

In sum, ICs occurred even when one or both of the letters appeared outside the 'attended region' bounded by the digits. This is not necessarily inconsistent with the attentional spotlight's view that ICs occur when both stimuli appear within an attended region-it is possible that participant's attentional spotlight occasionally spread outside the range of the cued digit target locations. However, further exploration of these data revealed more surprising results.

In order to understand IC effects more fully, we examined IC rates as a factor of either target or distractor position. Surprisingly, this analysis revealed that while the target letter's accuracy and rate of conjunction errors were strongly affected by the target letter's position relative to its neighboring digit, they were unaffected by the distractor letter's relative 
position $^{3}$. As we describe in the following paragraphs, target accuracy was reduced, conjunction errors were more frequent, and the IC effect was enhanced when the target stimulus appeared inside the position of the nearby digit rather than just outside it. Target accuracy and the occurrence of conjunction errors did not vary significantly with the position of the distractor letter, nor did the IC effect differ when examining trials where the distractor appeared inside versus outside its neighboring digit.

As noted previously, prior studies utilizing this experimental design predicted that ICs would occur when the target and distractor both fall within an attended region. It is thus surprising that IC rates were not significantly affected by distractor position. We discuss this further below and in the General Discussion.

Figure 2 and Table 3 display the impact of target versus distractor positions. Collapsing across distractor positions, we find that accuracy was significantly lower when the target letter appeared just inside the position of the digit rather than just outside it, $\mathrm{t}(17)=3.3$ and 4.7 for small and large spotlight conditions, $p<0.005$. Conjunction errors were likewise higher when the target letter was just within the digit's position rather than outside it, $\mathrm{t}(17)=2.9$ and $1.9, p<0.05$ and $p<0.10$ for the small and large spotlight conditions, respectively, and we found a corresponding higher estimated IC rate for the small spotlight condition when the target letter was inside the digit's position, $\mathrm{t}(17)=2.3, p<0.05$, though not for the large spotlight condition, $\mathrm{t}(17)=0.4, p>0.10$.

Though color errors were also elevated when the target letter was inside the digit's position, $\mathrm{t}(17)=1.9$ and $3.9, p<$ 0.10 and $p<0.005$, for the small and large spotlight conditions, respectively, the IC effect was significant in this experiment only when the target appeared between the two digits. That is, for the small spotlight, the IC effect was significant only when the target appeared at the innermost position, $t(17)$ $=3.4, p<0.005$, while the IC effect for the large spotlight was significant for targets appearing in the innermost position and marginally significant for target stimuli in the middle position from fixation, $\mathrm{t}(17)=3.2$ and $2.0, p<0.01$ and $p<0.10$, respectively. We term this asymmetry in target accuracy and ICs when the target letter is inside rather than outside the digit's position the inner-outer effect. In contrast, we did not find any inner-outer effect for the distractor letter (Table 4). Accuracy, conjunction errors, and color errors did not differ significantly when the distractor letter appeared within versus

\footnotetext{
${ }^{3}$ For consistency with Cohen \& Ivry (1989), results reported up to this point only used trials in which digits were correctly reported. Further results in this and later experiments will include all trials irrespective of digit report accuracy. These additional trials did not artificially inflate the IC effect-if anything, a $\chi^{2}$ test of independence found that digit-incorrect trials actually had a lower proportion of IC errors to color errors than digit-correct trials, $\chi 2(1, N=535)=$ $3.57, p<0.10$. Furthermore, incorporating the digit-incorrect trials did not substantially alter which positions exhibited significant IC effects.
}

outside of the digit's position, for either the small or large spotlight conditions, $\mathrm{t}(17)<1.5, p>0.10$.

It was interesting to us that the inner-outer effect differed between the small and large spotlight conditions. We used a series of repeated-measures two-way ANOVAs to examine the interaction between digit eccentricity and the inner-outer effect (target just inside versus just outside the digits) for target accuracy, color errors, conjunction errors, and the estimated IC rate, respectively. For accuracy, we found a significant main effect for digit eccentricity $(\mathrm{F}(1,17)=5.5, p<0.05)$ and the inner-outer effect $(\mathrm{F}=21.0, p<0.005)$ but no interaction between the two factors $(\mathrm{F}=2.3, p>0.10)$. An analysis of color errors found a significant main effect for the innerouter effect $(\mathrm{F}=19.3, p<0.005)$, and marginally significant effects for digit eccentricity $(\mathrm{F}=4.4, p<0.10)$ and the interaction between digit eccentricity and the inner-outer effect $(\mathrm{F}$ $=4.1, p<0.10$ ). That is, color errors were marginally higher in the large spotlight condition, and the inner-outer effect was marginally enhanced.

For conjunction errors, the inner-outer effect was significant $(\mathrm{F}(1,17)=8.6, p<0.01)$ but neither digit eccentricity nor the interaction effect rose to significance $(\mathrm{F}<1, p>0.10)$. The estimated IC rate did not have any significant main effect or interaction effects (digit eccentricity: $\mathrm{F}=0.5$; inner-outer effect: $\mathrm{F}=2.8$; interaction: $\mathrm{F}=2.5$; all $p>0.10$ ), though a onetailed analysis of the inner-outer main effect was marginally significant for the estimated IC rate, $p<0.10$.

In summary, our experiment both qualified and partially replicated the findings of Cohen and Ivry (1989). While we replicated the basic finding of IC's occurring between distal target and distractor letters, we also discovered that it is only the target letter and not the distractor that needs to be placed between the primary-task digit stimuli. This finding is at odds with one common interpretation of Feature Integration Theory in which the distractor must join the target inside the spotlight of attention for illusory conjunctions to occur.

In light of these results, we wished to further explore the role of attention in distal illusory conjunctions. Experiment 2 follows the example of Treisman \& Schmidt (1982) to test whether attention to the competing-task digits is necessary for illusory conjunctions to be produced.

\section{Experiment 2}

Experiment 2 investigates the effect of the visual presentation of the stimuli without any competing task requirements. Experiments 4 and 5 in Treisman \& Schmidt (1982) previously found that the IC effect disappeared when the dual task requirements were removed from their experiments. However, this variable was confounded with a pre-cueing procedure such that the location of the target stimulus was indicated at the beginning of each trial. It is unclear whether 

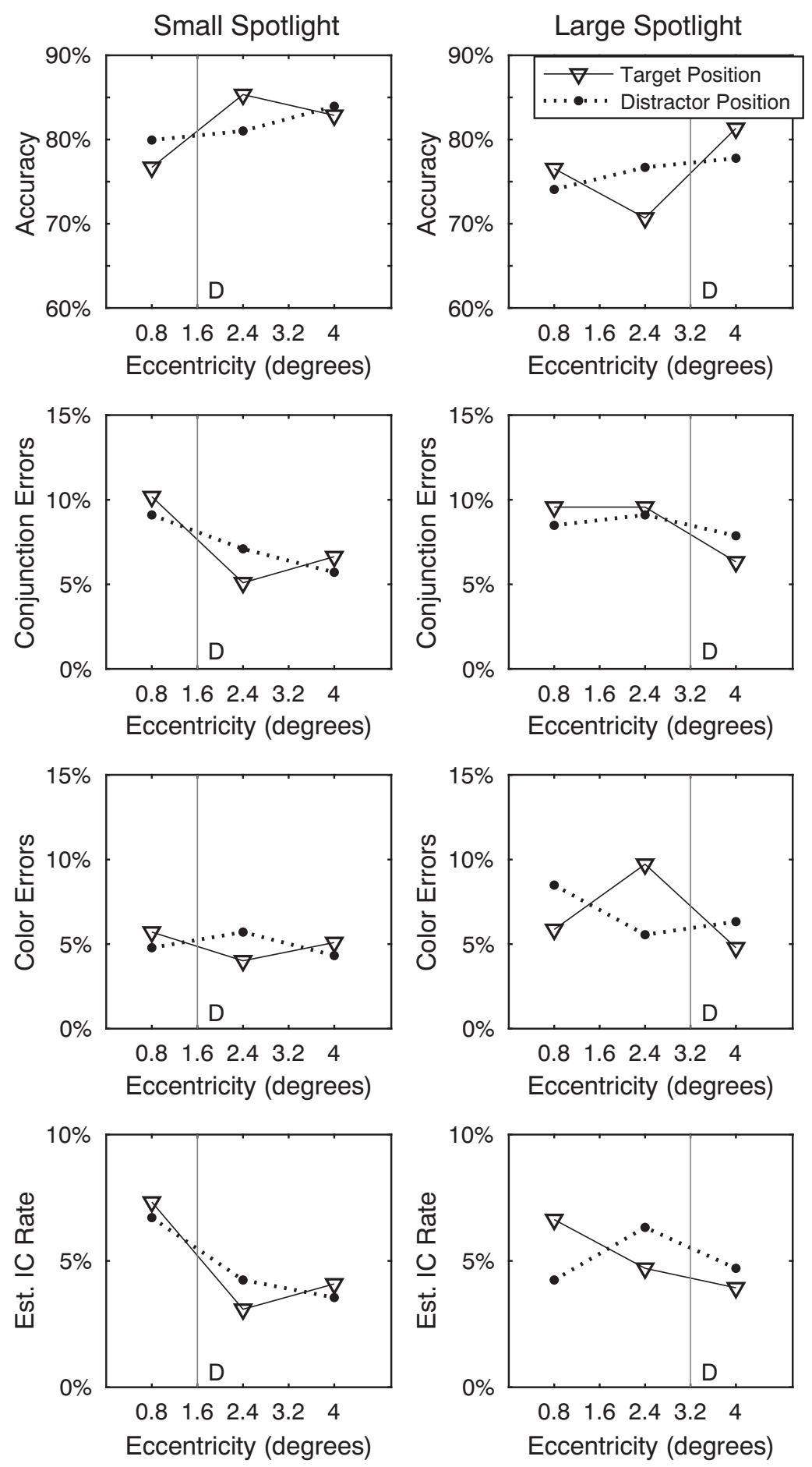

Fig. 2 Letter accuracy, conjunction errors, and color errors for Experiment 1. Data depicted here reflect the 18 main conditions of Experiment 1: (2 digit eccentricities) x (3 target letter eccentricities) $\mathrm{x}$ (3 distractor letter eccentricities). Data in the left column are from the small spotlight condition; data in the right column are from the large spotlight condition. Vertical lines indicate the digit position in each figure. The triangles with solid lines depict the data for different positions of the target letter, collapsed across distractor letter position; and the circles with the dotted line depict the results for different distractor letter positions, collapsed across the target positions. These

results demonstrate the inner-outer effect described in Experiment 1. Letter accuracy is reduced, and conjunction errors were enhanced when the target letters appears just inside the digit position relative to just outside the digit position for both the small and large spotlight conditions. The IC effect also showed a significant inner-outer asymmetry for the small but not the large spotlight condition. The distractor letter's position relative to the digit had no impact on any of these three measures. See also Table 3 for numerical comparisons and significance levels 
the loss of the IC effect is due to the removal of the competing task or whether it disappeared because participants could focus on only a single stimulus rather than several.

Furthermore, Treisman \& Schmidt's non-digit experiment removed the digit stimuli entirely rather than having participants ignore the digits. The visual presentation of a nearby, non-attended distractor has separately been found to impact proximal ICs (Henderson, 2014). Henderson (2014) found a strong relationship between proximal ICs and crowding, a phenomenon in which visual presentation of a distractor stimulus even when unattended could impair perception of a nearby target. The visual effect of nearby unattended digits is still unclear for distal ICs. Furthermore, it is worthwhile to examine how the visual presentation of the digits impacts the innerouter effect, not only for ICs but also for target accuracy.

Experiment 2 extended the procedure and materials of Experiment 1. Though the digits were still present, participants were instructed to ignore them and only report the color and identity of the target letter. Accuracy was matched across the two experiments by reducing the exposure duration and the contrast of the stimuli.

\section{Method}

Seventeen participants from the Stanford Paid Subject pool were paid $\$ 12$ for an hour-long experiment.

The stimuli and procedure in Experiment 2 were modified from that of Experiment 1 in three ways. First, participants were instructed that the digits were irrelevant. Second, to control for target accuracy between the two experiments, stimulus contrast was reduced for all of the colored letters, and luminance was reduced for the white stimuli (see Supplementary Materials). Third, stimuli were presented for a shorter period of time in Experiment 2, an average of $62.3 \mathrm{~ms}$ compared to $128.7 \mathrm{~ms}$ in Experiment 1, $\mathrm{t}(33)=9.44, p<0.001$. The shorter stimulus duration and reduced stimulus contrast were successful in controlling for target accuracy such that target accuracy did not significantly differ across the two experiments, $\mathrm{t}(33)=$ $0.251, p>0.10$.

\section{Results and discussion}

We tested whether the secondary digit task was necessary for the production of ICs or whether the visual presentation of digits was itself sufficient. Similar to Treisman \& Schmidt's (1982) non-digit experiment, Experiment 2 found no significant IC effect. In fact, we found marginally fewer conjunction errors than expected by our measure of random guessing, $\mathrm{t}(16)$ $=1.8, p<0.10$ (Table 2).

The lack of an IC effect in Experiment 2 corresponded to a change in the types of errors between Experiments 1 and 2. A two-way mixed-design ANOVA found a strong interaction for conjunction errors and color errors between experiments, with
Experiment 2 having relatively fewer conjunction errors and more color errors compared to Experiment 1, $\mathrm{F}(1,66)=10.3, p$ $<0.005$.

Since the colors of the target and distractor stimulus were mutually exclusive, the negative IC effect may be explained if participants noted and thereby excluded the distractor's color when guessing the target letter's color. If this is the case, Experiment 1 may have underestimated the IC effect. It is worth noting that Cohen \& Ivry (1989)'s Experiments 1 and 2 similarly found conditions in which there were fewer conjunction errors than expected by this measure of guessing, terming this phenomenon 'exclusionary feature errors'.

The removal of the digit task also eliminated the innerouter effect (see Table 3 and Fig. 3). If anything, there was marginally higher accuracy when the target stimulus appeared inside the position of the digits rather than outside it, perhaps due to a general loss of accuracy when targets appear further from fixation, $\mathrm{t}(16)=1.8, p<0.10$ (see e.g., Carrasco \& Frieder, 1997). Unlike phenomena like crowding, in which interference does not depend on attention to flanking stimuli, the inner-outer asymmetry in distal ICs appears to be mediated by participants either attending to or processing the digits.

Experiment 2's results argue that that some aspect of the digit task is responsible for the ICs in Experiment 1. However, it is difficult to rule out the potentially confounding roles of letter contrast and stimulus duration in the loss of an IC effect in Experiment 2. Tsal (1989) and Navon \& Ehrlich (1995) previously proposed that the short stimulus duration in Treisman \& Schmidt's (1982) non-digit experiment may have helped to eliminated their IC effect. For example, it is possible that the short durations led to increased guessing regarding the target's color, which would elevate both conjunction errors and color errors but eliminate any significant IC effect.

Arguing against this, we did not find an increase in conjunction errors in Experiment 2. Instead we found that conjunction errors had an absolute but non-significant decrease from an average of $7.9 \%$ responses in Experiment 1 to $4.2 \%$ in Experiment 2, $\mathrm{t}(33)=1.6, p>0.10$ (see Table 1). Regardless, the large reduction in stimulus contrast and duration in Experiment 2 remain topics of concern for the future.

Assuming the digit task drives ICs in Experiment 1, several questions remain regarding its precise role. The target letter is only reported after the digits in each trial; it is possible that the increased mnemonic demands of being reported last may lead to errors in which participants forget which letter had which color, leading to ICs. Experiment 3 reverses the response order of the digits and the target letter to examine their impact on the IC effect.

\section{Experiment 3}

Experiment 3 explored the role of the digit task and response order in the production of distal ICs. Tsal (1989) suggested 
Table 2 Comparison of estimated IC rates across Experiments 1-5

\begin{tabular}{|c|c|c|c|c|c|c|}
\hline Experiment & 1 & 2 & 3 & 4 & $5-\mathrm{D}$ & $5-\mathrm{L}$ \\
\hline Overall & $5.1 \% *(8.7 \%)$ & $-0.9 \% \sim(1.9 \%)$ & $1.4 \% \quad(3.9 \%)$ & $5.2 \% \ddagger(6.0 \%)$ & $8.6 \% \div(5.7 \%)$ & $5.4 \% \ddagger(6.0 \%)$ \\
\hline \multicolumn{7}{|c|}{ Small spotlight } \\
\hline $\mathrm{CD}$ & $9.5 \% \div(11.5 \%)$ & $1.0 \% \quad(6.5 \%)$ & $0.0 \% \quad(6.2 \%)$ & $5.7 \% *(8.4 \%)$ & $8.0 \% \div(8.2 \%)$ & $3.0 \% \quad(8.0 \%)$ \\
\hline $\mathrm{BD}-\mathrm{CE}$ & $5.7 \% *(8.6 \%)$ & $-0.2 \% \quad(4.4 \%)$ & $0.1 \% \quad(2.6 \%)$ & $3.6 \% \dagger(4.8 \%)$ & $7.3 \% \div(7.0 \%)$ & $3.5 \% \div(4.7 \%)$ \\
\hline $\mathrm{BE}$ & $1.6 \% \quad(9.3 \%)$ & $-0.7 \% \quad(8.7 \%)$ & $-1.2 \% \quad(3.6 \%)$ & $1.8 \% \sim(3.8 \%)$ & $2.5 \% \sim(5.3 \%)$ & $-0.5 \% \quad(5.5 \%)$ \\
\hline $\mathrm{AD}-\mathrm{CF}$ & $5.9 \% \sim(13.5 \%)$ & $-3.8 \% *(6.0 \%)$ & $-1.0 \% \quad(6.0 \%)$ & NA & NA & NA \\
\hline $\mathrm{AE}-\mathrm{BF}$ & $3.7 \% \sim(8.1 \%)$ & $-1.6 \% \quad(7.9 \%)$ & $-1.2 \%(4.3 \%)$ & NA & NA & NA \\
\hline AF & $1.9 \% \quad(8.0 \%)$ & $-2.2 \%(9.3 \%)$ & $-1.2 \% \quad(3.3 \%)$ & NA & NA & NA \\
\hline \multicolumn{7}{|c|}{ Large spotlight } \\
\hline $\mathrm{CD}$ & $5.3 \% \quad(13.3 \%)$ & $0.2 \% \quad(6.5 \%)$ & $2.5 \%(6.5 \%)$ & $5.6 \% \dagger(7.4 \%)$ & $8.6 \%+(7.0 \%)$ & $2.5 \% \sim(5.8 \%)$ \\
\hline $\mathrm{BD}-\mathrm{CE}$ & $5.6 \% *(8.6 \%)$ & $-1.6 \%(4.0 \%)$ & $2.5 \%(7.5 \%)$ & $4.7 \% \dagger(6.0 \%)$ & $7.7 \% \div(5.5 \%)$ & $6.8 \% \div(8.8 \%)$ \\
\hline $\mathrm{BE}$ & $7.2 \% *(12.5 \%)$ & $-0.2 \% \quad(9.3 \%)$ & $2.5 \%(9.0 \%)$ & $5.7 \% \dagger(7.0 \%)$ & $10.2 \% \$(10.2 \%)$ & $5.7 \% \div(6.9 \%)$ \\
\hline $\mathrm{AD}-\mathrm{CF}$ & $5.4 \% *(10.0 \%)$ & $1.1 \%(4.5 \%)$ & $-0.7 \% \quad(4.2 \%)$ & NA & NA & NA \\
\hline $\mathrm{AE}-\mathrm{BF}$ & $3.8 \% \quad(11.3 \%)$ & $-2.5 \% \quad(5.9 \%)$ & $0.6 \%(3.3 \%)$ & NA & NA & NA \\
\hline $\mathrm{AF}$ & $3.7 \% \quad(12.4 \%)$ & $-2.0 \% \quad(9.6 \%)$ & $2.5 \% *(4.4 \%)$ & NA & NA & NA \\
\hline
\end{tabular}

Note. Values are (Conjunction Errors $-0.5 \mathrm{x}$ Color Errors). Standard deviation values are in parentheses. Value includes both digit-correct and digitincorrect trials. 5-L = letter first, 5-D = digit first. $\sim$ : marginal significance, $*: p<0.05, \dagger: p<0.01, \uparrow: p<0.005$

that the competing task may produce ICs by providing an opportunity for forgetting or mnemonic interference. Competing task stimuli are reported prior to the target letter in many distal IC studies (e.g., Cohen \& Ivry, 1989; Treisman \& Schmidt, 1982; Tsal, Meiran, \& Lavie, 1994), and Tsal argued that this response order may lead to errors in target reporting either through delaying target responses (thereby allowing time for the decay of representations from the IC relevant stimuli) or by response interference produced by the earlier report of the digits.

We note that a simple mnemonic account does not explain Cohen \& Ivry (1989)'s and our results for ICs in certain positions but not others when mnemonic task demands were held constant. However, mnemonic interference or forgetting may play a role in the overall incidence of ICs in an experiment.
Navon \& Ehrlich (1995) explored the role of interference and memory decay in an IC matching task within a dual-task setting. Rather than describing a target stimuli, participants were asked whether a probe matched any of three critical stimuli present in a display. The probe could be identical to a stimulus; it could be a feature error (one feature from the stimuli present and one non-present feature); or it could be an IC error. Two digits flanked these stimuli on a portion of the trials, and Navon \& Ehrlich manipulated whether participants reported the digits before or after the probe.

Navon \& Ehrlich (1995) found more IC errors than feature errors, but they did not report any interaction between error type (IC versus feature) and response order, making it difficult to argue that reporting digits last reduces the IC effect. Several other results in Navon \& Ehrlich's experiment disagreed with

Table 3 Inner-outer effect based on target letter position across Experiments 1-5

\begin{tabular}{|c|c|c|c|c|c|c|}
\hline Experiment & 1 & 2 & 3 & 4 & $5-\mathrm{D}$ & $5-\mathrm{L}$ \\
\hline \multicolumn{7}{|l|}{ Small spotlight } \\
\hline Target letter accuracy & $-8.6 \% \ddagger(10.9 \%)$ & $5.1 \% \sim(11.3 \%)$ & $-5.6 \% *(9.2 \%)$ & $-4.2 \% \dagger(5.5 \%)$ & $-13.2 \% \div(11.3 \%)$ & $-9.0 \% *(16.5 \%)$ \\
\hline Conjunction report & $5.1 \%+(7.1 \%)$ & $-0.8 \% \quad(5.2 \%)$ & $0.6 \% \quad(2.0 \%)$ & $3.6 \% \dagger(4.4 \%)$ & $5.4 \% \ddagger(6.0 \%)$ & $2.2 \% \quad(6.2 \%)$ \\
\hline Color error & $1.7 \% \sim(3.7 \%)$ & $-3.4 \% \quad(8.1 \%)$ & $2.6 \% *(4.0 \%)$ & $0.2 \% \quad(2.5 \%)$ & $1.6 \% *(3.0 \%)$ & $-0.5 \% \quad(6.3 \%)$ \\
\hline Estimated IC rate & $4.2 \% *(7.5 \%)$ & $0.9 \% \quad(6.5 \%)$ & $-0.7 \% \quad(1.7 \%)$ & $3.5 \% *(4.8 \%)$ & $4.6 \% \ddagger(6.0 \%)$ & $2.5 \% \quad(7.5 \%)$ \\
\hline \multicolumn{7}{|l|}{ Large spotlight } \\
\hline Target letter accuracy & $-10.6 \% \$(9.4 \%)$ & $6.0 \% \sim(11.4 \%)$ & $-4.9 \%(12.7 \%)$ & NA & NA & NA \\
\hline Conjunction report & $3.2 \% \sim(6.9 \%)$ & $-1.0 \% \quad(4.8 \%)$ & $1.9 \% \quad(6.1 \%)$ & NA & NA & NA \\
\hline Color error & $4.9 \%+(5.2 \%)$ & $-3.9 \% \quad(10.4 \%)$ & $1.9 \% \sim(4.0 \%)$ & NA & NA & NA \\
\hline Estimated IC rate & $0.8 \% \quad(7.9 \%)$ & $1.0 \% \quad(6.9 \%)$ & $0.9 \% \quad(6.1 \%)$ & NA & NA & NA \\
\hline
\end{tabular}

Note. Standard deviation values are in parentheses. 5 - $\mathrm{L}=$ letter first, 5 -D = digit first. $\sim$ : marginal significance, $*: p<0.05, \uparrow: p<0.01,+: p<0.005$ 

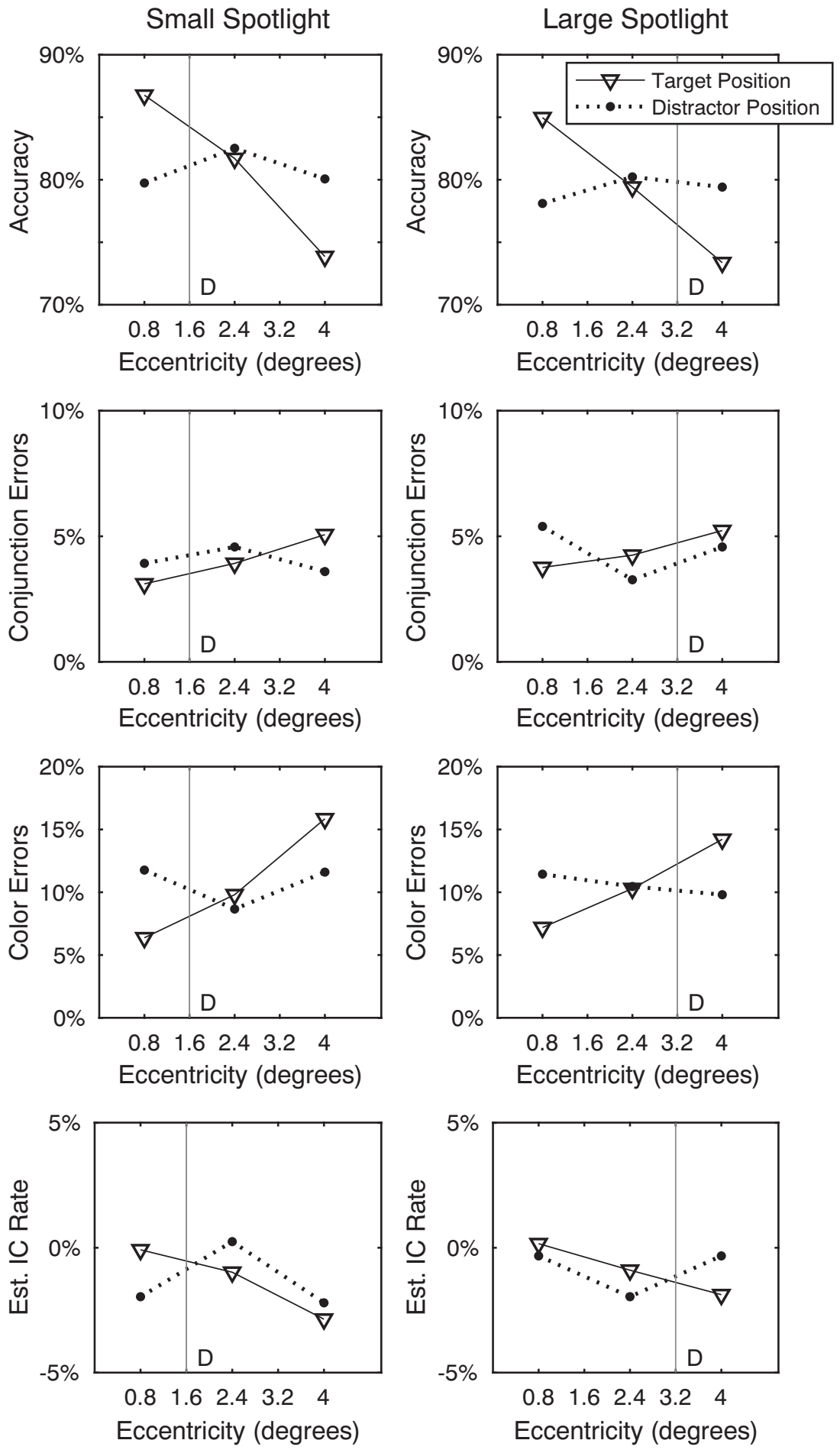

Fig. 3 Letter accuracy, conjunction errors, and color errors for Experiment 2, with data depicted using the format of Fig. 2. In the absence of a competing task, the inner-outer effect from Experiment 1

was eliminated. The inner-outer effect was marginally reversed for target accuracy, with higher accuracy when the target letter was inside the digit's position relative to outside its position

other studies' results, including our Experiment 2, making it difficult to draw firm conclusions. In particular, Navon \& Ehrlich found no interaction between error type and several manipulations including the presence or absence of the dual task. We believe it is possible the missing IC interaction with response order may be an effect of the matching task or another condition used in their experiment. Regardless, we find Navon \& Ehrlich's arguments on the importance of response order for ICs to be compelling, and we explore them further in Experiment 3. 
Experiment 3 switched the response order such that participants report the target letter prior to the digits. While this prevented mnemonic interference due to digit reports, it likely also de-prioritized the digit task despite instructions to the contrary. Experiment 3 thus examines the joint impact of task priority and mnemonic errors on ICs.

\section{Method}

Eighteen participants from the Stanford Paid Subject pool were paid \$12 for an hour-long experiment. All reported not being color blind.

The stimuli and procedure were adapted from Experiment 1. Participants were instructed to report the color of the target letter, the identity of the target letter, and then the two digits. However, participants were informed that reporting the digits was the more important task.

This experiment combines two pilot studies. Eight participants used an adaptive procedure with a threshold of 18/24 trials correct, while the remaining participants had a threshold of 22/24 trials correct. As a result, the two groups of participants differed in the duration of their digit+letter display. Participants with an 18/24 threshold had shorter stimulus durations $(\mathrm{M}=110.3 \mathrm{~ms}, \mathrm{SD}=41.2 \mathrm{~ms})$, compared to participants with a $22 / 24$ threshold $(\mathrm{M}=175.3 \mathrm{~ms}, \mathrm{SD}=41.3 \mathrm{~ms})$, $\mathrm{t}(16)=3.3, p<0.01$. The two groups of participants did not differ significantly in overall digit accuracy (85.1 and $81.5 \%)$, target accuracy ( 85.8 and $87.4 \%$ ), IC errors (3.6 and $1.7 \%$ ), or color errors $(4.6$ and $6.0 \%), \mathrm{t}(16)<1.7, p>0.10$. The two groups will be combined for further analysis.

\section{Results and discussion}

Experiment 3 examined the role of response order on the production of ICs. Based on Navon \& Ehrlich's (1995) arguments, we expected this change in response order to decrease ICs. Affirming this, our overall IC effect did not rise to significance in Experiment 3, $\mathrm{t}(17)=1.5, p>0.10$. There was generally no significant IC effect for each position (Table 2) even when positions were collapsed across distractor position.

This experiment involved a larger number of digit-incorrect trials in this experiment, which, in Experiment 1, had a notably lower ratio of IC-to-color errors than digit-correct trials. To remove this confound, we re-tested the data with only digitcorrect trials and still found no IC effect, $\mathrm{t}(17)=1.0, p>0.10$, nor did any additional positions demonstrate a significant IC effect.

Interestingly, Experiment 3 produced aspects of the innerouter effect that were visible in Experiment 1 but absent in Experiment 2 (Table 3; see also Fig. 4). Accuracy was lower when the target letter appeared just inside the digit's position than when it appeared just outside, for the small though not the large spotlight conditions, $\mathrm{t}(17)=2.5, p<0.05$ and $\mathrm{t}(17)=1.6$, $p>0.10$. This appears to have coincided with higher color errors rather than conjunction errors: conjunction errors did not change when the target letter was just inside versus outside the digit's position, $\mathrm{t}(17)=1.3$ and $1.2, p>0.10$ for the small and large spotlight conditions, but color errors increased significantly for the small spotlight condition, and this effect was marginally significant for the large spotlight condition, $\mathrm{t}(17)=$ 2.7 and $1.9, p<0.05$ and $p<0.10$, respectively. As with Experiment 1, the distractor letter's position relative to the digit had no impact on any of these three measures. One interpretation is that the digits influenced perception of the color of the target letter but not enough to produce ICs.

The comparison between Experiments 1 and 3 suggests that either mnemonic errors or task priority are critical for the production of IC errors. That is, changing the response order may have encouraged subjects to de-prioritize the digits during their initial display, reducing the impact of the dual task setting as well as the digits' ability to produce an inner-outer effect. We further address this question in Experiments 4 and 5. Experiment 4 increases the number of trials and adopts several other changes to improve statistical power. Experiment 5 then revisits the role of response order with participants post-cued about response order to preserve the same task priority between trials.

\section{Experiment 4}

Experiment 4 expanded on the methodology of Experiment 1 to increase statistical power. Experimental sessions increased to $90 \mathrm{~min}$ with a total of 384 test trials, and the outer letter positions were removed to concentrate trials on the inner four positions.

Two concerns from Experiment 1 were also addressed. Some responses may have been coded as conjunction errors when participants perceived only the colors of the letters and, by chance, paired the distractor's color with a correctly guessed letter identity. The number of potential letters was doubled to reduce the likelihood of participants guessing the letter identity on trials in which they only perceived the letters' colors. In addition, use of the same set of keys across all types of responses in Experiment 1 seemed problematic. Participants in Experiment 4 used different sets of keys for the different types of responses to reduce the likelihood of errors due to response overwriting from shared keys.

\section{Method}

Seventeen participants from the Stanford Paid Subject pool were paid $\$ 18$ for a 90 -min long experiment. One participant was previously in Experiment 1. All participants reported not being color blind.

The experimental stimuli changed in three ways. Two target letters were added, an ' $\mathrm{N}$ ' and an ' $\mathrm{S}$ '. The potential letter 

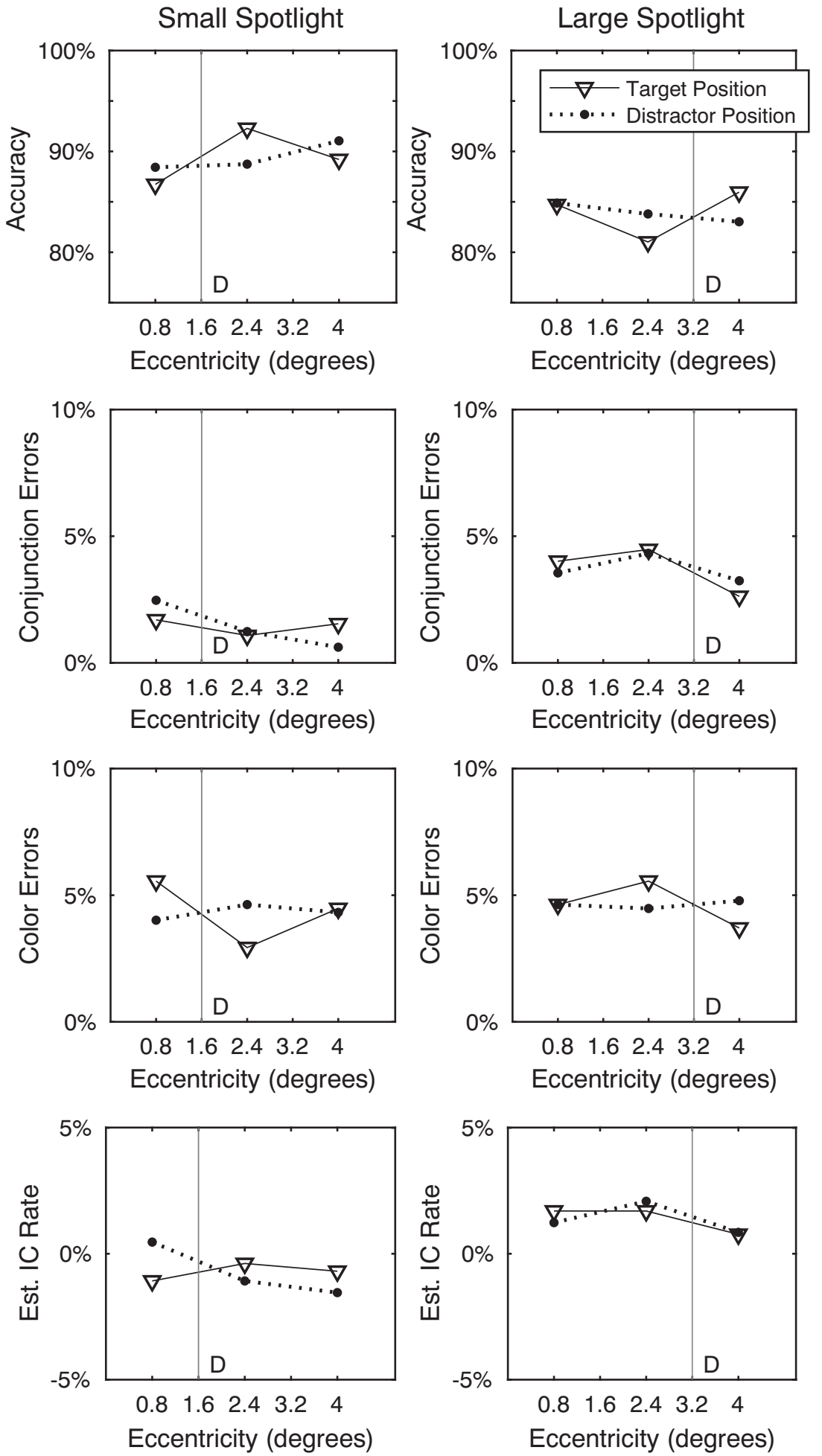

Fig. 4 Letter accuracy, conjunction errors, and color errors for Experiment 3, with data depicted using the format of Fig. 2. When participants reported the target letter's color and identity before reporting the digits, the inner-outer effect was substantially reduced. The inner-outer effect was marginally significant for target accuracy in the small but not large spotlight condition; significant for color errors in

positions were restricted to horizontal offsets of $0.8^{\circ}$ or $2.4^{\circ}$ from fixation. Lastly, the mask size increased to $8^{\circ} \times 16^{\circ}$.

the small spotlight condition and marginally significant in the large spotlight condition; and non-significant but in the correct absolute direction for conjunction errors in both spotlight conditions. The distractor letter's position relative to the digit had no impact on any of these three measures

The procedure largely matched Experiment 1 . The number of test trials increased to six blocks of 64 trials. Participants 
used keys 1-4 for digits responses; keys QWER to report the colors; and keys ASDF to report the target letter identity. Keys were labeled with the respective color or target letter. Lastly, the adaptive procedure was also slightly simplified (see Supplementary Materials).

\section{Results and discussion}

Results were generally similar between Experiments 1 and the current experiment. Digit accuracy and target accuracy were slightly higher than Experiment $1, \mathrm{t}(33)=2.3$ and 2.1, $p<$ 0.05 , and color errors were less frequent, $\mathrm{t}(33)=2.5, p<0.05$, though there was no significant difference in the IC error rate, $\mathrm{t}(33)=1.3, p>0.10($ see Table 1$)$.

Experiment 4 matched Experiment 1 in finding a significant overall IC effect, $\mathrm{t}(16)=3.5, p<0.005$. The proportion of IC errors and color errors did not change significantly between Experiments 1 and 4 after letter positions were controlled for: A mixed two-way ANOVA found no significant interaction between experiment and error type, $\mathrm{F}(1,60)=0.01, p>0.10$ (see also Table 2).

Likewise, Experiment 4 found significant IC effects in many of the same positions as Experiment 1. All four combinations of target-distractor positions exhibited a significant IC effect in the large spotlight condition, $\mathrm{t}(16)>2.5, p<0.05$. For these analyses, we separately examine conditions based on whether it was the target or the distractor letter which had greater eccentricity. In the small spotlight condition, we found a significant IC effect when the target letter appeared between the two digits, $\mathrm{t}(16)=2.7$ (distractor at $0.8^{\circ}$ ) and $\mathrm{t}(16)=3.5$ (distractor at $2.4^{\circ}$ ), $p<0.001$. There were additional marginally significant IC effects when the target appeared just outside the digit's position, $\mathrm{t}(16)=1.8$ (distractor at $0.8^{\circ}$ ) and 1.9 (distractor at $2.4^{\circ}$ ), $p<0.10$. These two marginal effects improved when collapsed across distractor position, such that we found a significant IC effect when the target appeared outside the digit's position, $\mathrm{t}=2.1, p<0.05$.

The inner-outer asymmetry found in Experiment 1 was strongly apparent in Experiment 4 (Fig. 5 and Table 3). Accuracy was significantly lower when the target letter appeared just inside the digit, $\mathrm{t}(16)=3.0, p<0.01$, and the estimated IC rate was higher when the target letter appeared inside the digit, $\mathrm{t}(16)=2.9, p<0.05$. Relatedly, a significant inner-outer effect was found for conjunction errors, $\mathrm{t}(16)=$ $3.2, p<0.01$, but not for color errors, $\mathrm{t}(16)=0.3, p>0.10$. As noted in Experiment 1, the inner-outer asymmetry only occurred for the target letter. Accuracy, ICs, and color errors did not differ based on the relative positions of the digits and distractor letters, $\mathrm{t}(16)<0.6, p>0.10$ (Table 4).

Many of our analyses have examined the effect of either target or distractor letter eccentricity while collapsing across the other letters' eccentricity. This allows us to control for the other variable's eccentricity. However, we also examined the inner-outer effect while holding the overall positions of letters constant and varying whether it was the target or the distractor letter that appeared outside the digits. We compared positions BD-CE for the small spotlight based on whether it was the target or the distractor which was inside the digits' position. We found significantly lower accuracy when the target was between the digits $(\mathrm{M}=-4.7 \%, \mathrm{SD}=6.7 \%, \mathrm{t}(16)=-2.9, p<$ $0.05)$, significantly higher conjunction errors $(\mathrm{M}=3.2 \%$, SD $=4.2 \%, \mathrm{t}(16)=3.1, p<0.01)$, and a significantly higher estimated IC rate $(\mathrm{M}=3.1 \%, \mathrm{SD}=4.0 \%, \mathrm{t}(16)=3.1, p<$ $0.01)$. Color errors did not differ significantly between conditions $(\mathrm{M}=0.2 \%, \mathrm{SD}=3.2 \%, \mathrm{t}(16)=0.3, p>0.10)$.

The IC effect and the inner-outer effect both remained after the number of target letters was doubled, reducing the likelihood that ICs were the results of guessing the target letter on trials in which neither letter was identified. Experiment 4 demonstrates that response overwriting due to shared keys was also unnecessary for either effect.

Experiment 5 utilized the procedure from Experiment 4 to further test the role of response order in a post-cue paradigm.

\section{Experiment 5}

Results from Experiment 3 and Navon \& Ehrlich (1995) suggest a role for response order in distal ICs. However, it is unclear whether the effect of response order on ICs was mediated by mnemonic errors, as Tsal (1989) argued, or by changes in the relative task priority of the digits and letters. Experiment 5 signaled the response order at the end of each trial, while maintaining digit priority both by instruction and by cuing digits first on $80 \%$ of trials. Experiment 5 thus investigates the role of report order on ICs when task priority is held constant.

\section{Method}

Twenty participants from the Stanford Paid Subject pool were paid $\$ 18$ for participation in a 90-min long experiment. All reported not being color blind.

Experiment 5 extended the stimuli and procedures of Experiment 4. Stimuli were supplemented with white arrow cues encompassing $0.4^{\circ} \times 0.2^{\circ}$ that could point either up or down. These white arrows appeared after the mask at $0.8^{\circ}$ above and below the former position of stimuli to prompt its report, while the fixation mark reappeared during the response period as a reference point. The arrows did not move to the next stimulus until the correct type of response (digit or letter) had been made.

When participants were cued to report the digits, these cues appeared above and below the positions of both digits simultaneously. Participants were able to freely choose which digit to report first. At this time, the trial would not proceed until two digit responses were entered. When participants were 

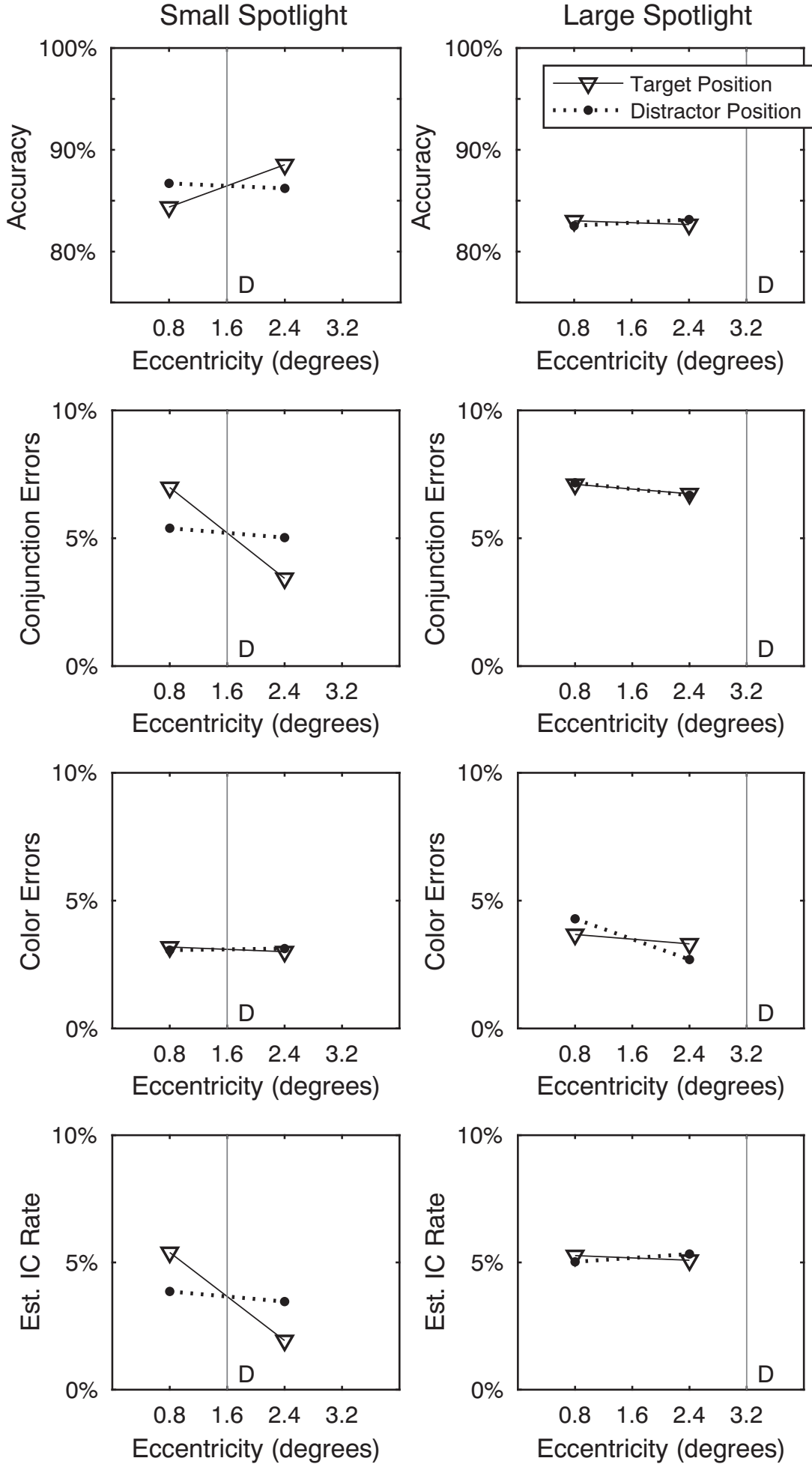

Fig. 5 Letter accuracy, conjunction errors, and color errors for Experiment 4. Data are depicted using the format of Figs. 2, 3 and 4, but reflect the eight main conditions of Experiment 4: (2 digit eccentricities $) \times(2$ target letter eccentricities $) \times(2$ distractor letter eccentricities). Due to the reduced number of target letter positions, the inner-outer effect could only be measured in the small spotlight condition.

Similar to Experiment 1, there was a significant inner-outer effect for target accuracy, conjunction errors, and the IC effect. We did not find a significant inner-outer effect for color errors in this experiment. As with prior experiments, the distractor letter's position relative to the digit had no impact on target accuracy, conjunction errors, color errors, or the IC effect 
Table 4 Inner-outer effect based on distractor letter position across Experiments 1-5

\begin{tabular}{|c|c|c|c|c|c|c|}
\hline Experiment & 1 & 2 & 3 & 4 & $5-\mathrm{D}$ & $5-\mathrm{L}$ \\
\hline \multicolumn{7}{|l|}{ Small spotlight } \\
\hline Target letter accuracy & $-1.1 \%(9.6 \%)$ & $-2.8 \%(0.056)$ & $-0.3 \%(7.7 \%)$ & $0.5 \%(4.0 \%)$ & $0.1 \%(8.9 \%)$ & $0.5 \%(9.9 \%)$ \\
\hline Conjunction report & $2.0 \%(5.6 \%)$ & $-0.7 \%(4.8 \%)$ & $1.2 \%(3.7 \%)$ & $0.4 \%(2.9 \%)$ & $0.9 \%(2.3 \%)$ & $0.7 \%(4.8 \%)$ \\
\hline Color error & $-0.9 \%(6.3 \%)$ & $3.1 \%(6.2 \%)$ & $-0.6 \%(4.2 \%)$ & $-0.1 \%(2.6 \%)$ & $-0.1 \%(7.8 \%)$ & $-0.5 \%(5.0 \%)$ \\
\hline Estimated IC rate & $2.5 \%(6.0 \%)$ & $-2.2 \%(6.4 \%)$ & $1.5 \%(5.2 \%)$ & $0.4 \%(3.6 \%)$ & $0.9 \%(4.6 \%)$ & $1.0 \%(5.0 \%)$ \\
\hline \multicolumn{7}{|l|}{ Large spotlight } \\
\hline Target letter accuracy & $-1.1 \%(10.3 \%)$ & $0.8 \%(11.4 \%)$ & $0.8 \%(12.3 \%)$ & NA & NA & NA \\
\hline Conjunction report & $1.2 \%(6.9 \%)$ & $-1.3 \%(5.3 \%)$ & $1.1 \%(4.3 \%)$ & NA & NA & NA \\
\hline Color error & $-0.8 \%(6.7 \%)$ & $0.7 \%(10.6 \%)$ & $-0.3 \%(5.0 \%)$ & NA & NA & NA \\
\hline Estimated IC rate & $1.6 \%(8.5 \%)$ & $-1.6 \%(8.1 \%)$ & $1.2 \%(3.5 \%)$ & NA & NA & NA \\
\hline
\end{tabular}

Note. Standard deviation values are in parentheses. $5-\mathrm{L}=$ letter first, $5-\mathrm{D}=$ digit first

cued to report on the target, the cues similarly appeared above and below the positions of both letters, and the trial would not proceed until a color and letter (X, F, N, or S) were entered.

Participants were cued to report the two digits first on $80 \%$ of trials in order to maintain the relative importance of the digits. Participants reported the target letter and then the two digits on the remaining $20 \%$ of trials. Participants were instructed that only these two types of cue orders existed. Participants were told that the digit task was their priority regardless of the response order.

Each participant had five test blocks of 80 trials each.

\section{Results and discussion}

Experiment 5 investigated whether mnemonic errors due to response order drove ICs when the task priority of the target stimulus was held constant. We found that changing the report order did not eliminate the IC effect given that task priority had been controlled. Both the digit-first and the letter-first conditions had significant overall IC effects, $\mathrm{t}(19)=6.5$ and $4.0, p<0.005$, arguing against memory interference and response delay as a full explanation for distal ICs.

Similar to Experiment 4, we found significant IC effects for almost all positions of the digit-first condition, across both small and large spotlight conditions, $\mathrm{t}(19)>4.2, p<0.005$ (see also Table 2). The sole exception was a marginally significant effect in the small spotlight condition with the target and distractor at $2.4^{\circ}$ offset from fixation (position BE), $\mathrm{t}(19)$ $=2.0, p<0.10$.

The letter-first condition had slightly fewer positions with significant IC effects: The target and distractor at the inner position (CD) was only marginally significant in the large spotlight condition, $\mathrm{t}=1.9, p<0.10$, and not significant in the small spotlight condition, $\mathrm{t}=1.6, p>0.10$ (Table 3 ). The IC effect was also not significant in the small spotlight condition when target and distractor were at $2.4^{\circ}$ offset (BE). This may have been due to the low number of trials per position for the letter-first condition: When the positions were collapsed across distractor positions, all positions yielded significant IC effects, $\mathrm{t}>2.5, p<0.05$. We found IC effects in similar positions for both response orders ${ }^{4}$.

Letter-first and digit-first conditions likewise both yielded an inner-outer effect (Fig. 6). For the digit-first condition, target position relative to digits had a highly significant effect on letter accuracy, conjunction errors, and estimated IC rates, $\mathrm{t}(19)=5.1,3.9$, and 3.3, $p<0.005$, as well as a significant effect on color errors, $\mathrm{t}(19)=2.3, p<0.05$. Target letter reports in the letter-first condition were significantly more accurate when they appeared outside the digit's position than inside, $\mathrm{t}(19)=2.4, p<0.05$. Though conjunction errors and the estimated IC rate were numerically greater for inside compared to outside target positions, the effect was non-significant, $\mathrm{t}(19)=$ 1.6 and $1.5, p>0.10$, possibly due to higher accuracy and a low number of trials (see Table 1). Similar to previous experiments, accuracy, ICs, and color errors did not demonstrate an inner-outer effect for distractor position for either the letterfirst or the digit-first conditions, $\mathrm{t}(19)<1.0, p>0.10$ (Table 4).

Interestingly, although we did not find an interaction between response order and color versus conjunction errors in our earlier ANOVA results, further exploration found that response order had a strong impact on the inner-outer effect, including conjunction errors and the estimated IC rate. Repeated-measures two-way ANOVAs explore the interaction between response order and the inner-outer effect for target accuracy, color errors, conjunction errors, and the estimated IC rate, respectively. Nearly all main and interaction effects were significant. For accuracy, response order, the inner-outer

\footnotetext{
${ }^{4}$ A lingering concern from this experiment is that the prevalence of digit-first trials may have left participants relatively unprepared for letter-first trials. The total response time for the four responses was substantially longer in the letterfirst than the letter-last condition, $\mathrm{M}=15.1 \mathrm{~s}(\mathrm{SD}=3.2 \mathrm{~s})$ versus $11.5 \mathrm{~s}(\mathrm{SD}=$ $2.8 \mathrm{~s}), \mathrm{t}(19)=10.0, p<0.001$, opening the possibility that letters reported in the letter-first condition may have been affected by some delay and possibly mnemonic interference.
} 

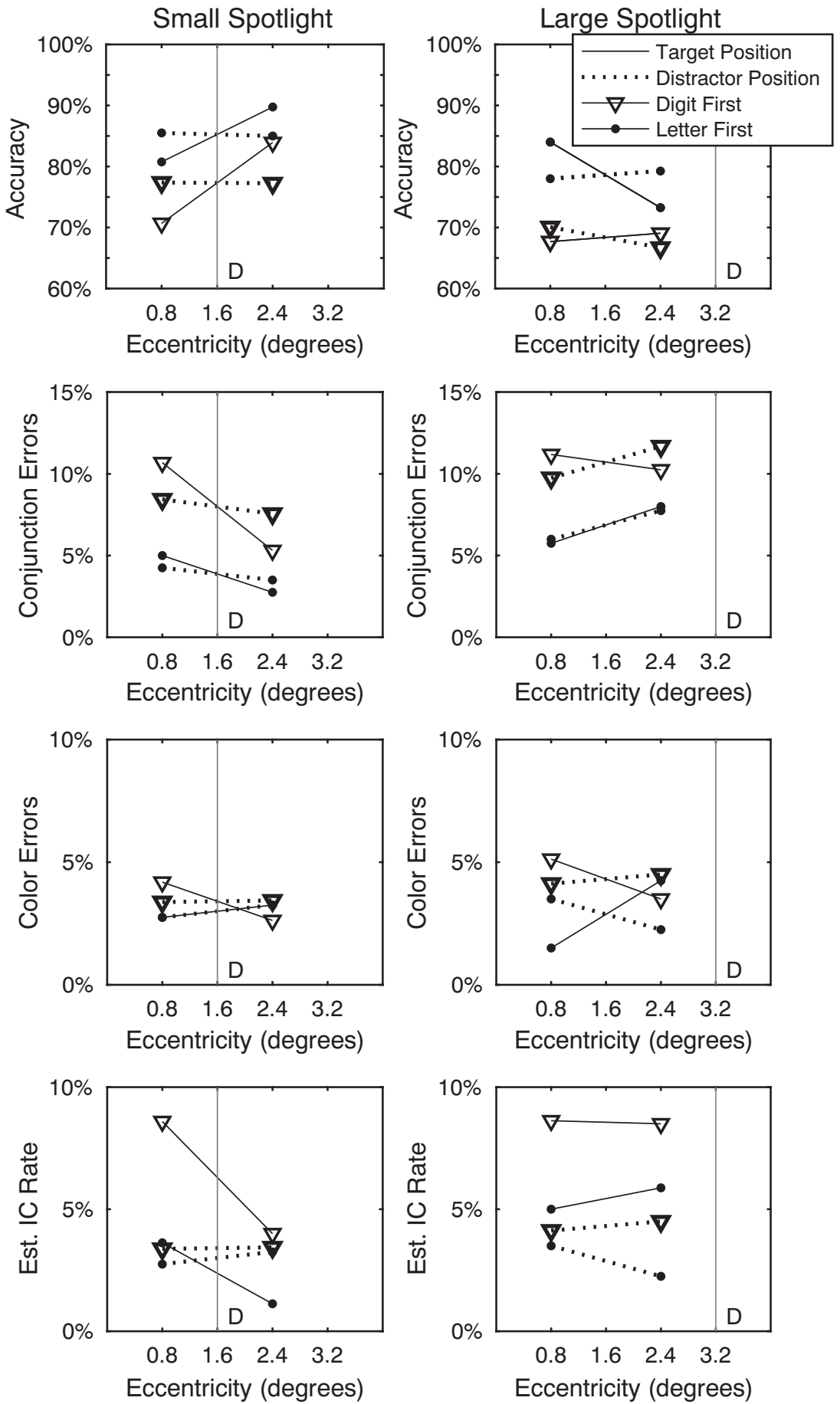

Fig. 6 Letter accuracy, conjunction errors, and color errors for Experiment 5. Data reflects the 16 main conditions of Experiment 5: (2 digit eccentricities) $\mathrm{x}$ ( 2 target letter eccentricities $) \times(2$ distractor letter eccentricities) $\mathrm{x}$ ( 2 response orders). Data in the left column are from the small spotlight condition; data in the right column are from the large spotlight condition. Vertical lines indicate the digit position in each figure. Solid lines depict the data for different positions of the target letter, collapsed across distractor letter position; dotted lines depict the data for different positions of the distractor letter, collapsed across target letter position. Lines with solid circle markers represent data from the

letter-first trials, while lines with upside-down triangles are from digitfirst trials. In digit-first trials, the inner-outer effect was significant for target accuracy, conjunction errors, color errors, and the IC effect. Letter-first trials found a significant inner-outer effect for target accuracy, and absolute but not significant inner-outer effects in conjunction errors, color errors, and the IC effect, possibly due to the low number of letter-first trials. The distractor letter's position relative to the digit had no impact on target accuracy, conjunction errors, color errors, or the IC effect 
effect, and their interaction were all highly significant, all F's $(1,19)>22, p<0.005$. Conjunction errors and the estimated IC rate had similar highly significant effects, all F's $(1,19)>$ $9, p<0.01$. Color errors alone did not show strong effects; while the main effect of response order was significant $(\mathrm{F}=$ 12.3, $p<0.005)$, the inner-outer effect and the interaction effect were only marginally significant, $F=3.8$ and 4.3 , respectively, $p<0.10$. Overall, these results suggest that reporting the digit first diminished accuracy, enhanced the IC effect, and also enhanced the inner-outer effects for target accuracy and ICs.

In summary, we did not find the digit-first response order was necessary for the general IC effect or the inner-outer effect; however, it does appear reporting the digits first enhanced these effects. We notes that it is unclear from these results whether the enhanced IC effect and inner-outer effects in the digit-first condition were the result of mnemonic interference or forgetting rather than a secondary effect of lowered target accuracy. The absence of IC effects in Experiment 3 likely resulted from a combination of participants prioritizing the report of the letters and their colors, together with the fact that they always reported the target letter before the digits.

\section{General discussion}

Our experiments replicated the distal illusory conjunctions effect, established a previously undiscovered spatial pattern driving ICs, and further clarified the role of the competing digit task. We first consider the role of the competing digit task before turning our attention to the other findings.

Within certain interpretations of our experimental paradigm, particularly that of Feature Integration Theory (FIT), ICs occur when the digit task manipulates a spotlight of attention to encompass both target and distractor letters, preventing participants from attending to the target by itself (Treisman \& Gelade, 1980; Treisman \& Schmidt, 1982; Cohen \& Ivry, 1989). Others have challenged this view of the digit task's role: Tsal (1989) and Navon \& Ehrlich (1995) argued that, if the digit task is indeed necessary for distal ICs, distal ICs may have a mnemonic rather than perceptual cause, arising when the digit reports delay or interfere with target letter reports. It is also possible that some aspect of the visual presentation of the digits affected the production of ICs.

Experiments 2-5 addressed these issues. In Experiment 2, distal ICs were eliminated in the absence of the digit task; visual presentation of the digit stimuli without a digit task was insufficient to product an IC effect. In Experiment 3, we found that ICs were eliminated when we changed the response order such that the target letter was reported prior to the digits. However, it was not clear if the ICs were eliminated because we had removed mnemonic errors or because we had inadvertently de-emphasized the digit stimuli. Experiment 5 used post-cues to direct response order after the visual stimuli were removed. We found that distal ICs still occurred when digits were prioritized while the letters were reported first, arguing that distal IC errors are not primarily mnemonic in nature.

A protagonist of a mnemonic account might still argue that items are encoded from a form of memory in priority order, such that by the time the participant manages to encode the target letter identity and color, information about it is no longer available. Such an account would have to explain how the inner-outer effect would arise in the form of memory proposed. We prefer instead to interpret the results in terms of the attentional shadow account we offer below.

Beyond manipulations to explore the role of the digit task, our experiments provide new evidence about the role of target and distractor location in producing ICs. Target accuracy and illusory conjunctions were strikingly affected by the relative positions of the target letter and its neighboring digit. Target accuracy was reduced and illusory conjunctions were increased when the target appeared inside rather than outside the position of the digit relative to fixation. We term this the inner-outer asymmetry effect. We found no such inner-outer effect for the distractor letter in our experiments. We note that the inner-outer effect is not due to the mere visual presence of the digits, since it did not appear in our Experiment 2 and was weak at most in Experiment 3; it occurred most clearly when the digits were attended to and prioritized. Here we argue that the inner-outer effect provides important clues about distal ICs, and may also shed light on the possible mechanisms and constraints involved in object recognition during the processing of displays with multiple stimuli.

Our findings contradict the idea that distal ICs only occur when the distractor stimulus joins the target stimulus within the spotlight of attention. If ICs are dependent on both stimuli falling within the spotlight of attention, then the IC effect should have been impacted by whether the distractor appeared between the digits, just as it was when the target appeared between them. As such, the idea from FIT that ICs occur among elements within the spotlight does not easily explain why the recipient of an IC (target stimulus) should depend on its location relative to digits but the source of the feature giving rise to the IC (distractor stimulus) should not. Instead, our results suggest that attention to or encoding of the digit may cast a sort of attentional 'shadow' that reduces accuracy for stimuli appearing within a limited area mostly lying to the foveal side of the digit's position, allowing for the intrusion of information from other positions. In Fig. 7 we provide a visual illustration of this idea.

An interesting feature of our findings is that the source of the intrusion was equally likely to be inside as well as outside the position of the digits. This seems to suggest that the shadow we are describing does not reduce the availability of color information for use in creating an illusory perception. The shadow seems instead to interfere with the proper integration 


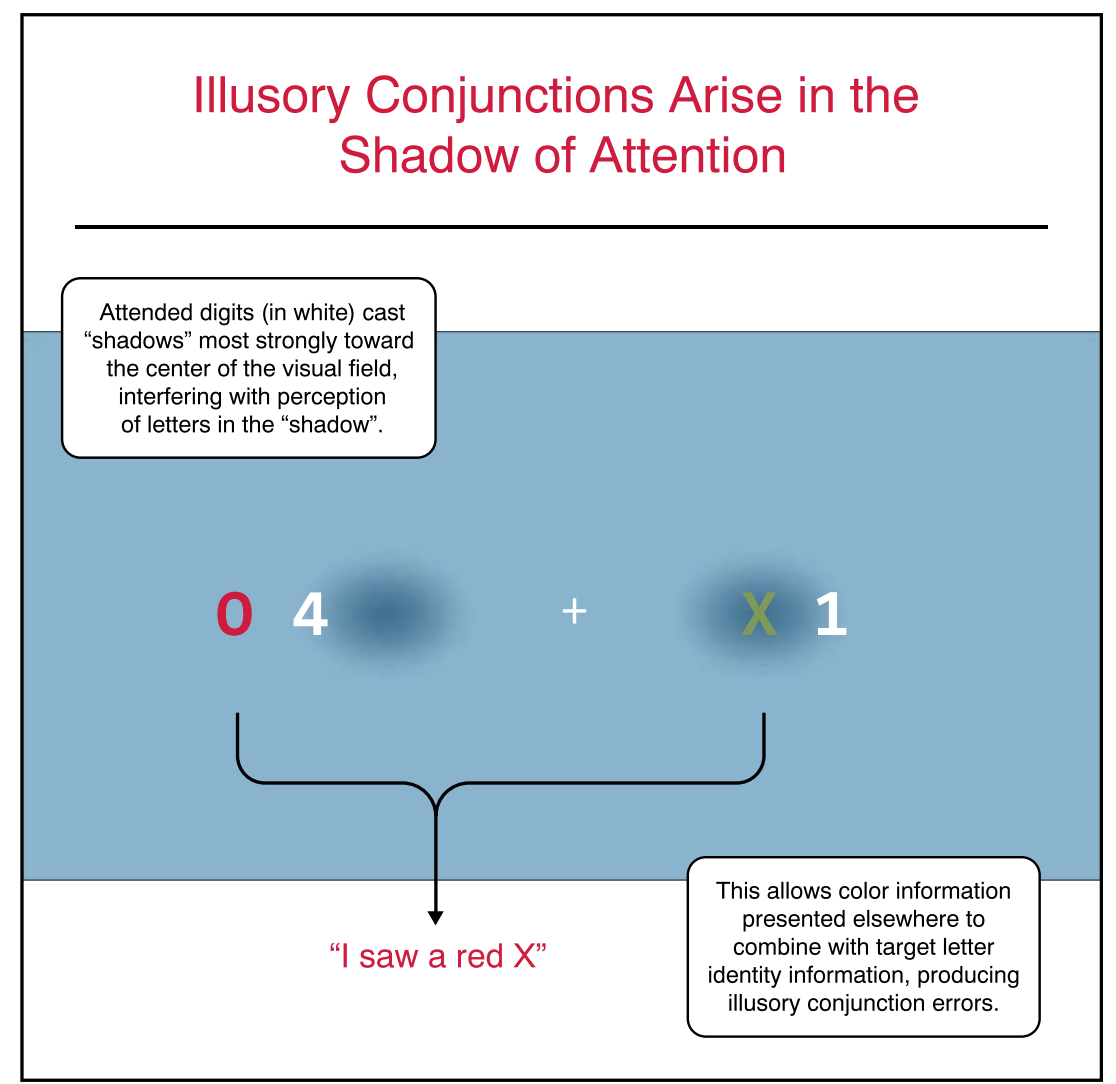

Fig. 7 Schematic illustrating potential regions with enhanced susceptibility to illusory conjunctions. This study finds greater susceptibility to illusory conjunctions when target stimuli appear just inside the position of an attended digit (dark blue spots), an effect we term the "shadow" of attention. The exact shape and breadth of this region

of letter and color information from the same position, while still allowing color information to be available for illusory perception at other positions.

These results do not contradict all aspects of FIT. On a broad level, FIT argues that ICs occur when a participant has been prevented from focusing separately on the target stimulus, and the conditions under which ICs occur in our experiment may indeed be conditions in which attention to the target is reduced. To capture the inner-outer effect, however, this sort of account would have to explain why participants would have lower accuracy on target letters between digits than on target letters outside of the digits. We prefer the idea, then, as captured in Fig. 7, that attention to one stimulus (in our case, the prioritized digits), produces interference with perception of other stimuli (in our case, the target letter) inward from the focus of attention.

Our results suggest that the mechanisms by which FIT or other theories explain distal ICs may need to be re-examined. For FIT, rather than driving an attentional spotlight, the digit closest to the target stimulus appears to interfere in some way with the formation of an integrated percept of the target. An adaptation of FIT to these results might argue that the attended digit serves to block participants' ability to shift attention to is unclear. We note that illusory conjunctions were also found outside the shadowed region, possibly indicating a baseline level of illusory conjunctions across the display (see Experiment 5 in the Supplemental Materials). The distance between letters and digits is altered from the experiments for display purposes

the target stimulus and so render it susceptible to ICs. The possible implications this could have for attentional interactions on visual perception are, we believe, themselves interesting and worthy of further study.

However, there are several other results that, combined with the current study, lead us to rethink the general form of FIT. FIT has been supported by three main empirical findings: proximal ICs, distal ICs, and ICs produced by patients with Balint's syndrome, a neurological disorder in which patients are seemingly impaired in their ability to correctly perceive multiple stimuli presented at the same time. These results have been taken as support for the strong version of FIT in which unimpaired perception only occurs when a single stimulus has been attended and perceived at a time. However, we believe that several results, including those we report here, argue for a re-examination of these three main empirical findings underlying FIT.

We argue that the human visual system is capable of simultaneously processing a few stimuli without interference under normal circumstances (Henderson \& McClelland, 2011; Henderson, 2014), as long as the stimuli are not too close to each other and attention is not directed elsewhere. Regarding proximal ICs, we have separately found evidence that 
proximal ICs can be largely or entirely explained by crowding (Henderson, 2014). Regarding distal ICs, the results we have presented in the current paper suggest that these ICs occur by a different mechanism than has previously been presented in FIT and the attentional spotlight theory, and regarding ICs in patients with Balint's syndrome, we have previous presented a computational simulation of a visual system able to simultaneously process a small number of stimuli in parallel. We found this model to be able to accommodate the effects of Balint's syndrome on perception of multiple stimuli (Henderson \& McClelland, 2011; Henderson, 2014; Robertson et al., 1997). This finding argues that Balint's syndrome is not immutable evidence that normal visual object recognition occurs for a single stimulus at a time. Altogether, we believe that the three main findings supporting FIT can be reinterpreted in a way consistent with the view that the human visual system can accurately process multiple arbitrary stimuli in parallel, but within limits imposed by proximity, attention, and other factors. In short, we do not believe that proximal IC's, distal IC's and Balint's syndrome necessarily require us to accept the view that we must shift attention between individual stimuli to accurately bind each stimulus' features together into an integrated percept.

These views coincide with neurophysiological findings in macaques when viewing small numbers of stimuli. Neuronal responses to multiple, briefly presented stimuli demonstrated an averaging effect suggesting concurrent rather than sequential processing (V2 \& V4: Reynolds, Chelazzi, \& Desimone, 1999; IT: Zoccolan, Cox, \& DiCarlo, 2005).

A softer theory partially consistent with FIT might include concurrent visual processing of small numbers of stimuli. Instead of holding that correct feature binding always requires strictly sequential attention to each separate item in a display, a softer version of the theory could propose that under certain constraining circumstances - strong dual task demands, an overload of stimuli, or a nearby attended digit—it is possible that this normal parallel (concurrent) object processing breaks down, and we are instead in the situation described by FIT, where features from stimuli separated widely in visual space can be integrated to form an illusory percept. We leave it to future researchers to more fully explore the parameters of this space.

While we focus on the role of a nearby digit in driving ICs, it did not appear to be the sole factor capable of producing distal ICs. We also found ICs in Experiment I when the target was outside of the digit's position and relatively distant. An IC effect was additionally present in a sixth experiment (see Supplementary Materials), when the digits were placed along the vertical axis from fixation and so were distant from the horizontally aligned letters. Aspects of the distal IC paradigm may result in a nonzero baseline level of ICs, and investigating the prerequisites for this effect might be a helpful goal to further understand ICs.
Overall, we find that distal ICs are highly dependent on a particular set of conditions. In contrast to proximal ICs (Henderson, 2014), distal ICs occur under brief stimulus durations and a competing task, and they are sensitive to the importance of the competing task stimuli. Proximity between the target and distractor stimulus did not seem to drive our distal ICs: stimuli appeared in opposite hemifields, and the lack of any inner-outer effect for distractor letter location implies that target-distractor proximity did not enhance ICs under these conditions (in contrast to findings for IC rates in proximal IC studies, see e.g., Ashby et al., 1996).

Instead, the competing task stimulus was particularly effective in driving distal ICs for nearby target stimuli, with decreased accuracy, increased conjunction errors, and increased ICs when the target was inside the position of the competing task stimulus relative to fixation. That the relative position of the distractor letter did not seem to matter is difficult to explain within the attentional spotlight version of FIT. While interference from the competing task stimuli does not appear to be the only source of distal ICs in this paradigm, we believe this interference is indicative of an interesting new phenomenon by which local, possibly attention-driven interference can lead to long-range visual effects.

We had several remaining questions from these results. Here we highlight two of them. First, what is the spatial extent in which a nearby digit affects the processing of a target stimulus? The digit's effect on the target may have been superimposed on a general eccentricity effect (e.g. Fig. 3). Unfortunately, we did not vary the target's position enough to find the boundary at which the digit ceased to affect the target, nor is it clear if the digit also increased ICs for a small region outside its position or above and below its position.

Second, to what extent does attention to a digit modulate the inner-outer effect? When digits were unattended in Experiment 2, the IC effect completely disappeared as did the inner-outer effect on target accuracy. When attention to the digits was potentially reduced in Experiment 3 (compared to Experiment 1), the IC effect disappeared but there were still traces of an inner-outer effect on target accuracy. Our results were suggestive that the degree of attention to the digits affected the inner-outer effect; further investigation is needed to explore this possibility.

The answers to these two questions would provide clues towards the cognitive and neural mechanisms underlying illusory conjunctions and the inner-outer effect. How and in what neural region does attention to the digit interfere with the target letter's representation such that it is susceptible to interference? How do features for a distant distractor stimulus in the opposite visual hemifield affect the target? And lastly, the position of a nearby digit had a strong impact on perception of the target's features. Why does the position of the nearby digit appear to have no impact on the availability of the distractor's features for conjunction errors? We hope that our work has 
opened up an opportunity to explore these questions further, and that these explorations will shed more light on the binding problem and the means by which our visual system identifies and distinguishes objects in a cluttered visual world.

Acknowledgements The work reported here was conducted while the first author was a PhD student in the Department of Psychology at Stanford University. We thank William Prinzmetal for encouragement and valuable comments.

Data and materials for the experiments reported here are available by request. None of the experiments were preregistered.

\section{References}

Ashby, F.G., Prinzmetal, W., Ivry, R., \& Maddox, W.T. (1996). A formal theory of feature binding in object perception. Psychological Review, 103(1), 165- 192.

Bouma, H. (1970). Interaction effects in parafoveal letter recognition. Nature, 226, 177-178.

Carrasco, M., \& Frieder, K.S. (1997). Cortical magnification neutralizes the eccentricity effect in visual search. Vision Research, 37(1), 6382.

Chastain, G. (1982). Feature mislocalizations and misjudgments of intercharacter distance. Psychological Research, 44, 51- 66.

Cohen, A., \& Ivry, R. (1989). Illusory conjunctions inside and outside the focus of attention. Journal of Experimental Psychology: Human Perception and Performance, 15(4), 650- 663.

Henderson, C.M. (2014). The binding problem and the perception of multiple stimuli Doctoral dissertation, Stanford University. https:// searchworks.stanford.edu/view/10608091.

Henderson, C. M. \& McClelland, J. L. (2011). A PDP model of the simultaneous perception of multiple objects. Connection Science, 23, 161- 172 .

Ivry, R.B., \& Prinzmetal, W. (1991). Effect of feature similarity on illusory conjunctions. Perception \& Psychophysics, 49, 105- 116.

Keele, S. W., Cohen, A., Ivry, R., Liotti, M., \& Yee, P. (1988). Tests of a temporal theory of attentional binding. Journal of Experimental Psychology: Human Perception and Performance, 14, 444- 452.

Navon, D., \& Ehrlich, B. (1995). Illusory conjunctions: Does inattention really matter? Cognitive Psychology, 29, 59- 83.
Pelli, D.G., Palomares, M., \& Majaj, N.J. (2004). Crowding is unlike ordinary masking: Distinguishing feature integration from detection. Journal of Vision, 4(12), 1136- 1169.

Pelli, D.G., \& Tillman, K.A. (2008). The uncrowded window of object recognition. Nature Neuroscience, 11(10), 1129- 1135.

Prinzmetal, W., Henderson, D., \& Ivry, R. (1995). Loosening the constraints on illusory conjunctions: Assessing the roles of exposure duration and attention. Journal of Experimental Psychology: Human Perception \& Psychophysics, 21 (6), 1362- 1375.

Prinzmetal, W., \& Keysar, B. (1989). A functional theory of illusory conjunctions and neon colors. Journal of Experimental Psychology: General, 118, 165-190.

Prinzmetal, W., \& Mills-Wright, M. (1984). Cognitive and linguistic factors affect visual feature integration. Cognitive Psychology, 16, 305340.

Prinzmetal, W., Treiman, R., \& Rho, S. H. (1986). How to see a reading unit. Journal of Memory and Language, 25, 461- 475.

Reynolds, J.H., Chelazzi, L., \& Desimone, R. (1999). Competitive mechanisms sub-serve attention in macaque areas V2 and V4. Journal of Neuroscience, 19, 1736- 1753.

Robertson, L., Treisman, A., Friedman-Hill, S., and Grabowecky, M. (1997). The interaction of spatial and object pathways: Evidence from Balint's syndrome. Journal of Cognitive Neuroscience, 9, 295-317.

Treisman, A., \& Gelade, G. (1980). A feature-integration theory of attention. Cognitive Psychology, 12, 97-136.

Treisman, A., and Schmidt, H. (1982). Illusory conjunctions in the perception of objects. Cognitive Psychology, 14, 107-141.

Tsal, Y. (1989). Do illusory conjunctions support the Feature Integration Theory? A critical review of theory and findings. Journal of Experimental Psychology: Human Perception and Performance, $15,394-400$.

Tsal, Y., Meiran, N., \& Lavie, N. (1994). The role of attention in illusory conjunctions. Perception \& Psychophysics, 55(3), 350-358.

Vickery, T.J., Mok Shim, W., Chakravarthi, R., Jiang, Y.V., \& Luedeman, R. (2009). Supercrowding: Weakly masking a target expands the range of crowding. Journal of Vision, 9(2):12, 1- 15.

Whitney, D., and Levi, D.M. (2011). Visual crowding: A fundamental limit on conscious perception and object recognition. Trends in Cognitive Science, 15(4), 160-168.

Wolford, G., \& Shum, K.H. (1980). Evidence for feature perturbations. Perception \& Psychophysics, 27, 409- 42.

Zoccolan, D., Cox, D.D., \& DiCarlo, J.J. (2005). Multiple object response normalization in monkey inferotemporal cortex. Journal of Neuroscience, 25(36), 8150-8164.

Publisher's note Springer Nature remains neutral with regard to jurisdictional claims in published maps and institutional affiliations. 